\title{
METHOD FOR ACCURATELY EVALUATING FLOW CAPACITY OF INDIVIDUAL FILM-COOLING ROWS OF ENGINE COMPONENTS
}

\author{
Benjamin Kirollos, Thomas Povey \\ Osney Thermofluids Laboratory, \\ Department of Engineering Science, \\ University of Oxford, Oxford, OX2 OES, UK \\ Email: ben.kirollos@eng.ox.ac.uk \\ Email: thomas.povey@eng.ox.ac.uk
}

\begin{abstract}
A laboratory experimental method and analysis technique is presented for evaluation of individual film-cooling row flow capacity characteristics. The method is particularly suited to complex systems such as hot section nozzle guide vanes (NGV) with lossy feed system characteristics. The method is believed to be both more accurate and more experimentally efficient than previous techniques.

The new analysis technique uses an experimentally calibrated network model to represent the complex feed system, and replaces the need for internal loss measurements, which are both experimentally demanding and inaccurate. Improvements in the experimental method include a differential mass flow rate measurement method (with a bypass flow) which improves repeatability and reduces experiment time.

We demonstrate the method using two high pressure (HP) $N G V$ designs: an engine part with relatively uncoupled (in terms of internal loss) cooling rows; and a laser-sintered part with highly coupled cooling rows. The HPNGV designs are experimentally assessed in both a laboratory calibration facility, and in an engine-realistic aerodynamic field. We show that the individual-row flow capacity can be evaluated in the calibration facility to a $2 \sigma$ precision of approximately $0.3 \%$. Errors associated to scaling to the engine aerodynamic field are estimated.
\end{abstract}

\section{INTRODUCTION}

Precise prediction of coolant mass flow through individual film cooling rows is necessary to accurately predict part cooling effectiveness, overall capacity [1], and engine efficiency [2]. Inaccurate evaluation leads to unoptimised performance, and can require cooling system re-design at a late stage in the engine development cycle.

The purpose of this paper is to present a rapid and accurate laboratory experimental technique to measure individual-row coolant mass flow rate- or coolant flow capacity - of engine-scale turbine vanes at engine conditions. The technique is equally applicable to real engine components and laboratory proxies (additive manufacturing), which are increasingly used for high fidelity experimental assessment.

The technique is well suited to the mass flow rate range encountered for HPNGVs at laboratory conditions, allowing individual-row flow capacity measurements (of HPNGV) to be measured typically in a single day to a $2 \sigma$ precision of $\sim 0.3 \%$. Laboratory experimental results are scaled to engine conditions using experimentally validated analytical tools which are described. Assessment of, for example, the onset of mainstream ingestion is possible using these tools.

This paper has three main sections. In the first section, the underlying theory, experimental facility (Coolant Capacity Rig, University of Oxford) and methods for measuring individualrow coolant flow capacity are described. In the second section, the flow capacity measurement method is demonstrated using a recent production-engine cast HPNGV (geometry $A$ ) in which the cooling system is relatively uncoupled (we discuss this subsequently), and a method for scaling to engine conditions is described and validated using a higher TRL facility (Annular Sector Heat Transfer Facility, University of Oxford). In the third section the method is demonstrated on a HPNGV with a highly coupled internal cooling system (geometry B).

We define coupled systems as those in which the cooling capacities (defined with respect to a system inlet total pressure and row exit static pressure) are interdependent because of internal losses. This means that the sum of individual row capacities, measured by blocking the row of interest, is not equal to the overall cooling capacity of the vane. In these systems, loss characteristics need to be experimentally evaluated to allow determination of individual row capacities and prediction of row capacity at the engine condition. The proposed method allows this to be done without intrusive instrumentation. We define uncoupled systems to be those in which rows interact only minimally. In these systems the sum of individual row capacities, measured by blocking the row of interest, is approximately equal to the overall vane cooling capacity.

A significant advantage of the new method is that it is applicable to both uncoupled and highly coupled systems, with no requirement for measurements (of total pressure loss) internal to the part. This simplifies the experimental procedure. The method can be used in situations where computational and empirical estimations of row flow capacity (typically based on discharge coefficient and internal loss correlations, and requiring geometric row areas) are not sufficiently accurate. 


\section{CAPACITY THEORY}

Mass flow rate is often represented using the term capacity

$$
\Gamma=\frac{\dot{m} \sqrt{T_{0}}}{p_{0}}
$$

where $p_{0}$ is total pressure, $T_{0}$ is total temperature and $\dot{m}$ is mass flow rate. For uniform one-dimensional compressible flow,

$$
\Gamma=A\left(\frac{p_{0}}{p}\right)^{-\frac{\gamma+1}{2 \gamma}}\left\{\frac{2 \gamma}{R(\gamma-1)}\left[\left(\frac{p_{0}}{p}\right)^{\frac{\gamma-1}{\gamma}}-1\right]\right\}^{1 / 2}
$$

Here, $p_{0} / p$ is the total-to-static pressure ratio, $A$ the effective area (the same as the actual area for an ideal system), and $\gamma$ and $R$ the ratio of specific heats and gas constant, respectively.

Capacity is a dimensional quantity (units of $\mathrm{kg} \mathrm{s}^{-1} \mathrm{~K}^{1 / 2}$ $\mathrm{Pa}^{-1}$ ), but the primary dependence of mass flow rate on $T_{0}$ and $p_{0}$ is removed, allowing the capacity characteristic (curve) to be evaluated. In ideal one-dimensional systems with fixed $A, \gamma$ and $R$, the characteristic $\Gamma=f\left(p_{0} / p\right)$ is known as the isentropic capacity characteristic. If we intend to compare ideal systems operating with different gases, we write $\Gamma=$ $f\left(p_{0} / p, \gamma, R\right)$.

In real systems the effective area is a function of $p_{0} / p$ and the coolant Reynolds number $\operatorname{Re}_{d}$ (e.g. [3]). We write

$$
\Gamma=f\left(\frac{p_{0}}{p}, \gamma, R, \operatorname{Re}_{d}\right) .
$$

In comparisons of capacity characteristics for the same parts between lab and engine conditions, $R$ is approximately matched for the two different cooling flows and the dependence on this can be ignored. For completeness, we have also ignored the impact of the metal temperature of the part, because the thermal expansion in the hot condition is a relatively small, and affects the vane throat area and cooling hole area by a similar amount.

\section{Coolant Capacity of Film-Cooled Turbine Vanes}

For the purpose of this paper, we categorise film-cooled turbine vanes as either:

(1) Coupled, indicating that the individual row cooling capacities (defined with respect to a system inlet total pressure and row exit static pressure) are interdependent because of internal losses; or

(2) Uncoupled, indicating that the rows interact only minimally.

When cooling rows are situated relatively far apart, and the internal loss inducing (duct loss) mechanisms result in only a small pressure drop (in comparison to the coolant-tomainstream total pressure difference, where leading edge rows are concerned), coupling is weak and the cooling rows can be considered uncoupled. In many vane systems, cooling rows are fed by a shared, relatively loss-inducing duct, however, and the coupling effect cannot be ignored. Both coupled and uncoupled systems can be treated using the methods described in this paper. We now deal with each in turn.

\section{Uncoupled system}

In an uncoupled system, eq. (3) can be applied to an individual cooling row:

$$
\tilde{\Gamma}_{i}^{r}=\frac{\widetilde{m}_{i}^{r} \sqrt{T_{0 c}}}{p_{0 c}}=f\left(\frac{p_{0 c}}{p_{\mathrm{ext}, i}^{r}}, \gamma_{c}, \operatorname{Re}_{d, i}\right)
$$

where $\tilde{\Gamma}_{i}^{r}$ is the coolant capacity of row $i, p_{0 c}$ and $T_{0 c}$ are the coolant total pressure and total temperature at vane inlet, $\widetilde{m}_{i}^{r}$ is the mass flow rate through row $i$ and $p_{\mathrm{ext}, i}^{r}$ is the exit static pressure (i.e., the local mainstream static pressure) into which row $i$ is ejected. (N.B. The tilde on $\tilde{\Gamma}_{i}^{r}$ represents the coolant capacity of row $i$ when all rows are blowing, in order to differentiate it from the capacity measurements $\Gamma_{i}^{r}$ reported in this paper. The distinction is important and will be discussed later).

\section{Coupled system}

In a coupled system, total pressures at the entrance to particular cooling rows are determined by internal losses, which depend on internal loss coefficients (we define them as $K_{A}^{c}, K_{B}^{c}, K_{C}^{c}$ etc.), and the entire flow established within the vane, which is a function of the individual cooling row capacities $\left(\tilde{\Gamma}_{1}^{r}, \tilde{\Gamma}_{2}^{r}, \tilde{\Gamma}_{3}^{r}\right.$ etc.). The coolant capacity of a particular row is - in principle, at least - dependent on the capacity of all other rows and all loss coefficients. We write

$$
\tilde{\Gamma}_{i}^{r}=f\left(\frac{p_{0 c}}{p_{\mathrm{ext}, i}^{r}}, \gamma_{i}, \operatorname{Re}_{d, i}, \tilde{\Gamma}_{1}^{r}, \tilde{\Gamma}_{2}^{r}, \tilde{\Gamma}_{3}^{r}, \ldots, K_{A}^{c}, K_{B}^{c}, K_{C}^{c}, \ldots\right),
$$

Of course, the dependence of one particular row on another may be weak (where they are fed from different plena for example). This is accounted for in the analysis that follows.

\section{Scaling between rig and engine conditions}

In the experimental determination of coolant capacity all relevant non-dimensional groups-eqs. (4) or (5) - can either be matched to engine conditions or accounted for using experimentally validated analytical tools (discussed later in the paper). To match coolant $\mathrm{Re}$ in the rig environment typically requires exhausting to an elevated (non-atmospheric) pressure, however, or using foreign (dense) gas, both of which are considerable experimental complications. Exhausting to atmosphere typically reduces coolant $\mathrm{Re}$ by a factor of 4.5 compared the engine. The impact is a coolant capacity (estimated from [3]) approximately 6\% lower than in the engine. This can be accounted for with a correction factor, a method which is believed to be robust since the Re mismatch affects all rows similarly (see [3]). Scaling to engine conditions is not the prime topic of this paper, but the correction factor method is believed by the authors to be adequate, and is mentioned here for completeness.

\section{COOLANT CAPACITY RIG}

The Coolant Capacity Rig (CCR) was designed and built to obtain fast, accurate and repeatable individual-row coolant capacity measurements of both cast and laser-sintered vanes. 
Typical individual-row capacity measurements of a HPNGV can be completed in a single day to a $2 \sigma$ precision of $\sim 0.3 \%$.

Novel aspects of the technique include: a differential mass flow measurement technique incorporating bypass flow; the requirement for only one calibrated mass flow nozzle which can cover all PRs; and minimising the number of rows that must be blanked to determine individual-row coolant capacity characteristics. These features are discussed in later sections. For turbine vanes with coupled cooling rows, we demonstrate that CCR measurements can also be used to estimate internal loss coefficients, without using internal pressure probes.

Photographs of the CCR are shown in Figs. 1 and 2 and a schematic shown in Fig. 3. Operating conditions are summarised in Tab. 1.

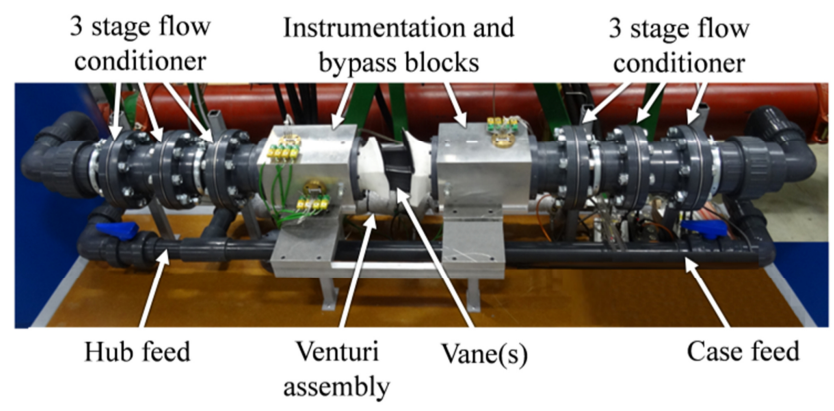

FIGURE 1. PHOTOGRAPH OF COOLANT CAPACITY RIG.

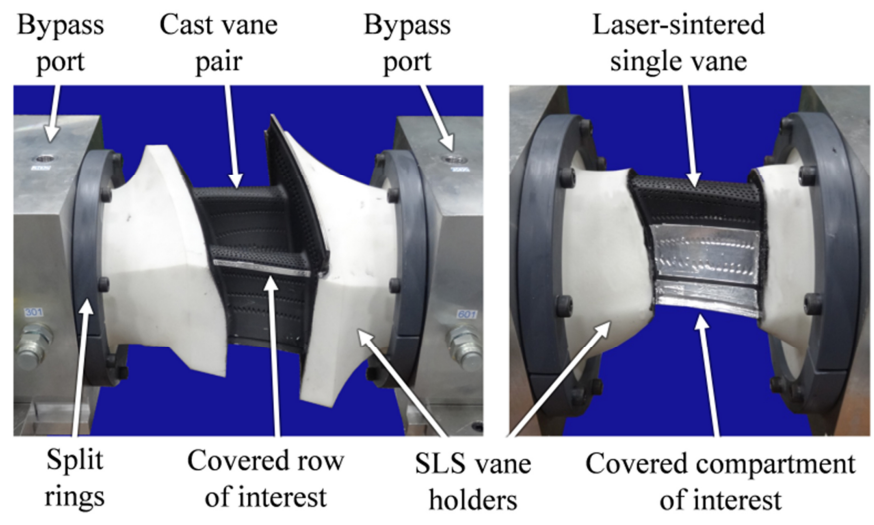

FIGURE 2. PHOTOGRAPHS OF VANES IN COOLANT CAPACITY RIG. (L) CAST PAIR; (R) LASER-SINTERED SINGLE.

TABLE 1. OPERATING CONDITIONS OF THE COOLANT CAPACITY RIG (CCR), MODEL POINTS WITH REFERENCE TO FIG. 3.

\begin{tabular}{lcc}
\hline \hline Parameter & Value & Units \\
\hline Upstream venturi meter $p_{0,1}$ & $2.5-5$ & bara \\
Downstream venturi meter $p_{0,2}$ & $1.05-1.2$ & bara \\
Vane inlet pressure $p_{0,3 \mathrm{~A}}$ and $p_{0,3 \mathrm{~B}}$ & $1.03-1.15$ & bara \\
Coolant total temperature & $\sim 290$ & $\mathrm{~K}$ \\
Coolant $\gamma_{\mathrm{CCR}}$ & 1.40 & \\
Mass flow rate & $0.015-0.025$ & $\mathrm{~kg} / \mathrm{s}$ \\
Coolant Re (based on nominal hole & $\sim 2000$ & \\
diameter $0.75 \mathrm{~mm})$ & & \\
\hline \hline
\end{tabular}

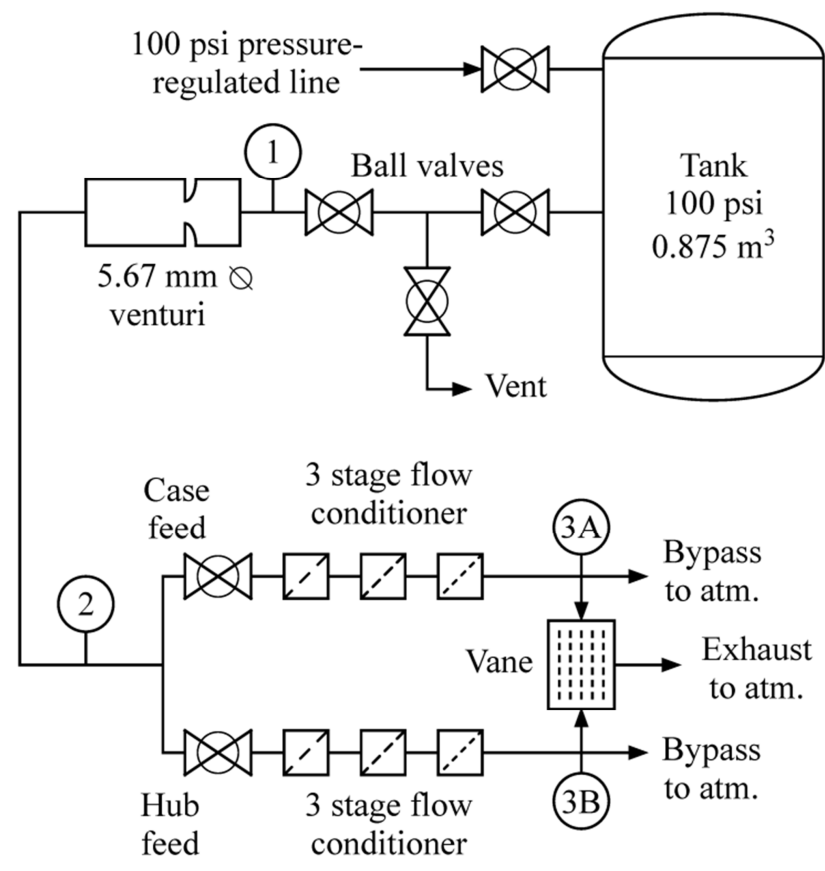

FIGURE 3. SCHEMATIC OF COOLANT CAPACITY RIG.

Flow is delivered from a $0.875 \mathrm{~m}^{3}$ air tank through a calibrated sonic mass flow measurement venturi, then through a threestage flow conditioner $(30 \%, 60 \%$ and $75 \%$ open area) to hub and case feeds of a test HPNGV (typically either single lasersintered vane or cast pair). Experiments are conducted in blowdown mode, so that a range of pressure ratios $p_{0 c} / p_{a t m}$ is covered in a single run, where $p_{0 c}$ is the total pressure at inlet to the vane (average of points $3 \mathrm{~A}$ and $3 \mathrm{~B}$ in Fig. 3 ) and $p_{a t m}$ is the ambient exhaust pressure. Total temperature and total pressure are measured upstream of the vane hub and case inlet using measurement rakes. Feed pipe diameters of 4" are used to reduce total pressure loss between the pressure measurement points (3A and 3B, Fig. 3) and the vane inlet. Bypass ports (see Fig. 2) are used to control the total mass flow rate through the system, to ensure the measurement nozzle remains within its calibrated range: this is discussed in detail subsequently. A static leak test verified that pipework leaks were at worst $0.005 \%$ of nominal coolant mass flow.

The vanes are interfaced with the instrumentation/bypass blocks using custom-made vacuum impregnated plastic selective laser-sintered (SLS) holders, as shown in Fig. 2. The seal between the plastic holders and instrumentation/bypass blocks is made good using face O-rings and split-ring clamps. The vanes are sealed into the plastic holders using silicone sealant. An alignment method is used to ensure this delicate seal is not unduly stressed, or subject to movement. Individual rows or compartments are selectively blanked using externally applied $3 \mathrm{M}$ aluminium foil tape (the purpose of this is discussed in detail later).

Mass flow rate in the CCR is measured using a choked venturi meter according to the equation: 


$$
\dot{m}=C_{D_{1}} \frac{\pi d_{1}^{2}}{4} \frac{p_{01}}{\sqrt{T_{01}}} \sqrt{\frac{\gamma}{R}\left(\frac{\gamma+1}{2}\right)^{-\frac{\gamma+1}{\gamma-1}}}, \quad \frac{p_{01}}{p_{2}}>1.89
$$

where $\dot{m}$ is the instantaneous mass flow rate through the venturi meter, $C_{D_{1}}$ is the discharge coefficient of the venturi meter, $d_{1}$ is the nominal throat diameter $(5.67 \mathrm{~mm}), D_{1}$ is the upstream pipe diameter $(25.4 \mathrm{~mm}), T_{01}$ and $p_{01}$ are the total pressure and total temperature upstream of the venturi meter (at position 1 , Fig. 3), $p_{2}$ is the static pressure downstream of the venturi meter (at position 2, Fig. 3), and $\gamma$ and $R$ have their normal meanings. The $5.67 \mathrm{~mm}$ venturi meter is designed in accordance with [4] for a cylindrical throat but without downstream divergent section. The discharge coefficient of the venturi meter was found as a function of Reynolds number through cross-calibration with a Flow Systems, CEESIcalibrated choked venturi nozzle with nominal diameter $d_{3}=8.28 \mathrm{~mm}$.

The instantaneous mass flow rate measured at the venturi meter (point 1, Fig. 3) is within $0.05 \%$ of the instantaneous mass flow rate at vane inlet (points $3 \mathrm{~A}$ and $3 \mathrm{~B}$, Fig. 3), estimated using the unsteady mass flow analysis in [5]. Consequently, the unsteady mass flow correction terms are neglected. The coolant capacity of the system (which incorporates all open film cooling rows and bypass ports) is evaluated using eq. (1), the mass flow rate at the venturi meter $\dot{m}$ and the total temperature $T_{03}$ and total pressure $p_{03}$ upstream of the vane (average of points $3 \mathrm{~A}$ and $3 \mathrm{~B}$, Fig. 3 ). The $2 \sigma$ precision error (repeatability) of a single absolute capacity measurement (of the entire system) was experimentally determined to be $0.015 \%$. Thus the $2 \sigma$ precision of an individual row measurement is $2 * 0.015 \% *$ total capacity/row capacity. The additional factor of two is due to the fact that a row measurement is the result of two experimental runs. Given an approximate value of total capacity / row capacity $=11$, the $2 \sigma$ precision of an individual row measurement is $\sim 0.3 \%$.

\section{DIFFERENTIAL MASS FLOW MEASUREMENT TECHNIQUE FOR SINGLE-ROWS}

To allow a single venturi meter to be used within its calibration range for all experiments, a differential mass flow measurement technique was used. Where the mass flow rate of a very low capacity outlet was to be assessed - a single row for exampleall other outlets (all other rows) were typically left open, and if necessary the total exit flow area was further increased by opening a bypass port so that the mass flow rate through the CCR venturi meter remained within the calibrated range. We refer to this experiment as a high-differential run. A second experiment is then performed in which only the outlet under investigation (the single row of interest, for example) is blocked. We refer to this as a low-differential run. The difference between the low- and high-differential runs is a function of the capacity of the row of interest. This differential approach has two advantages:

i) By leaving all rows open and using a bypass flow where necessary, a single calibrated venturi nozzle can be used for all experiments. This removes the additional complexity and cumulative uncertainty terms that arise when more than one nozzle is used.

ii) In this method, the degree of coupling in the system is as representative as possible of the undisturbed system to be characterized. That is, internal losses are as closely replicated as possible, requiring smaller correction terms than would otherwise be necessary.

We now illustrate the overall technique with an example.

Figure 4 shows the experimental steps to evaluate the capacity of a single row of interest. A high-differential run is performed across a range of pressure ratios, and the measured capacity characteristic of the entire system, $\Gamma_{H}\left(p_{0 c} / p_{a t m}\right)$, determined. A low-differential run is performed, and a second measured capacity characteristic determined, $\Gamma_{i, L}^{r}\left(p_{0 c} / p_{a t m}\right)$. The difference between these tests is blanking of the row of interest, and thus the difference in the measured capacity characteristics is a function of the capacity characteristic of that row. We write:

$$
\Gamma_{i}^{r}\left(\frac{p_{0 c}}{p_{a t m}}, \gamma_{a}\right)=\Gamma_{i, H}^{r}\left(\frac{p_{0 c}}{p_{a t m}}, \gamma_{a}\right)-\Gamma_{i, L}^{r}\left(\frac{p_{0 c}}{p_{a t m}}, \gamma_{a}\right)
$$

where $\gamma_{a}$ is the ratio of specific heats at ambient room temperature.

It proves convenient to group the ambient (a) nondimensional parameters:

$$
\Pi_{a}=\left\{\frac{p_{0 c}}{p_{a t m}}, \gamma_{a}\right\}
$$

in order to simplify the equations in this paper, e.g., eq. (7) becomes:

$$
\Gamma_{i}^{r}\left(\Pi_{a}\right)=\Gamma_{i, H}^{r}\left(\Pi_{a}\right)-\Gamma_{i, L}^{r}\left(\Pi_{a}\right)
$$

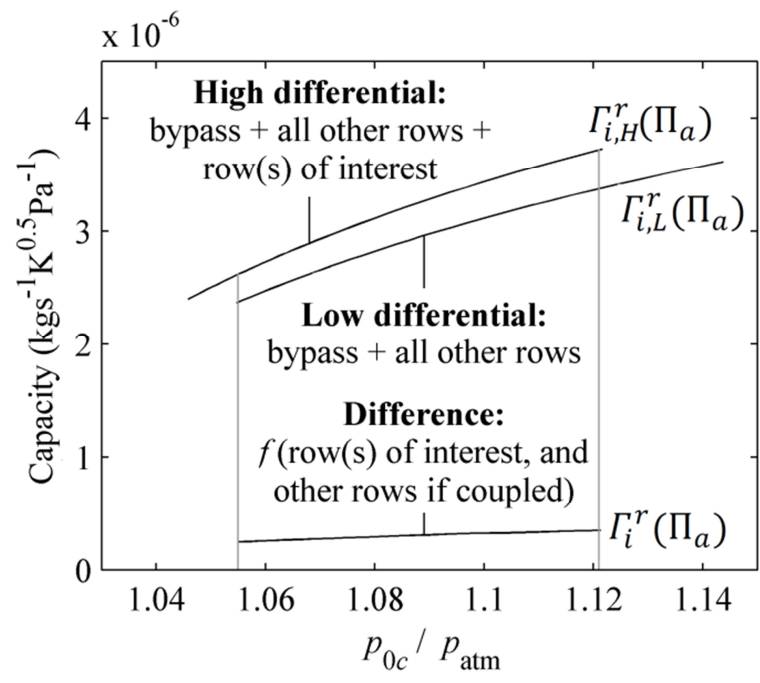

FIGURE 4. DIFFERENTIAL MASS FLOW MEASUREMENT TECHNIQUE: ROW CAPACITY MEASUREMENT $\Gamma_{i}^{r}\left(\Pi_{a}\right)$; HIGHDIFFERENTIAL SYSTEM CAPACITY MEASUREMENT $\Gamma_{i, H}^{r}\left(\Pi_{a}\right)$; LOWDIFFERENTIAL SYSTEM CAPACITY MEASUREMENT $\Gamma_{i, L}^{r}\left(\Pi_{a}\right)$ WITH ROW OF INTEREST BLOCKED. 


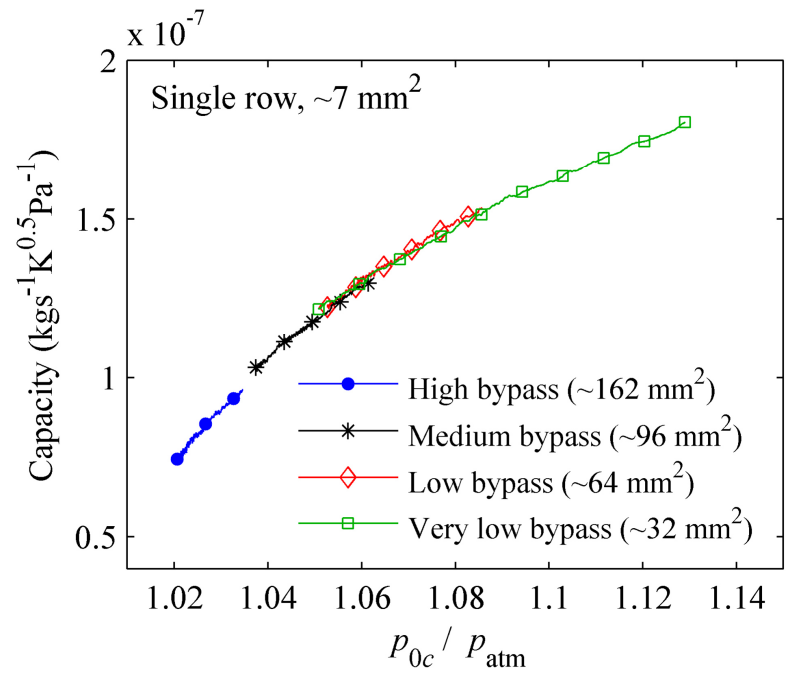

FIGURE 5. CAPACITY MEASUREMENT OF A SINGLE COOLING ROW $\Gamma_{i}^{r}\left(\Pi_{a}\right)$ DETERMINED FROM DIFFERENTIAL MEASUREMENTS USING FOUR DIFFERENT BYPASS FLOW SETTINGS.

It is worth noting that the bypass capacity characteristic does not need to be determined in this method. The bypass flow is therefore set to a convenient value to keep the nozzle within calibration range.

Where a capacity characteristic is desired over a very wide range of pressure ratios, a number of different bypass flow settings may be required to keep the venturi meter within calibration range. This is illustrated in Fig. 5 which shows the result of differential measurements using four different bypass flow settings, for a single cooling row. Excellent collapse onto a common capacity trend is achieved without knowledge of the individual bypass capacity characteristics, despite the order-ofmagnitude difference between the cooling row capacity (equivalent to an area of $7 \mathrm{~mm}^{2}$ ) and the bypass nozzle capacities (equivalent to areas between $32 \mathrm{~mm}^{2}$ and $162 \mathrm{~mm}^{2}$ ). It is notable that this occurs across a pressure ratio range from $1.02<p_{0 c} / p_{\text {atm }}<1.14$.

An identical technique can be used to perform capacity measurements of an entire $j^{\text {th }}$ compartment $\Gamma_{j}^{c}\left(\Pi_{a}\right)$ (for vanes with multiple feed compartments), and entire vane capacity $\Gamma^{t}\left(\Pi_{a}\right)$. In both cases the bypass flows are chosen to keep the venturi flow meter within range across the range of pressure ratios under investigation. The technique allows comparatively small capacity trends (single row trends, for example) and comparatively large capacity trends to be measured with good accuracy across a wide range of pressure ratio without the requirement for multiple nozzles (which implies multiple calibrations, nozzle changes, and compound uncertainty).

In summary, the differential measurement technique with bypass flows has the following advantages:

i) Only a single measurement venturi meter is required, which can be kept within calibration range whist investigating both comparatively small and large capacity characteristics over a wide range of pressure ratios. This eliminates compound uncertainty due to multiple nozzles, and considerably simplifies the experimental procedure. ii) Typically, only the rows/compartment under investigation is blanked, leading to closer similarity of the internal coupling of rows between rig and engine situations. Although the coupling terms can be treated as a correction, using methods described later in this paper, improving similarity in the underlying experiments further reduces error terms.

There are two additional practical advantages which reduce the chance of experimental error:

iii) The requirement to blank rows is minimised since only the row/compartment of interest is blanked. This minimises the risk of a leak forming in rows which are intended to be blanked.

iv) The differential technique is independent of system leaks.

\section{ANNULAR SECTOR HEAT TRANSFER FACILITY}

In the CCR, all rows exhaust to atmospheric pressure. In the engine, rows exhaust to different pressures, which depend on the mainstream Mach number distribution. A method which allows prediction of the engine condition, which has different internal coupling from the CCR condition, is presented later in this paper. The scaling method is validated using capacity measurements made in the University of Oxford Annular Sector Heat Transfer Facility (Sector Facility). The facility is now described.

The Sector Facility is a transonic, non-rotating, pressureregulated, annular sector facility which replicates enginerealistic (Take-off or Cruise) conditions of $\mathrm{M}$, mainstream Re, $p_{0 c} / p_{0 m}$ and Tu. Real engine cast parts (HPNGVs) or lasersintered counterparts can be tested. The Sector Facility coolant Reynolds number is $\sim 3000$ (based on a nominal cooling hole diameter of $0.75 \mathrm{~mm}$ ) and is approximately double the coolant Reynolds number in the CCR. The coolant flow is air $(\gamma=1.4)$ at ambient temperature and the mainstream flow is air at $\sim 70$ ${ }^{\circ} \mathrm{C}$. The test section comprises an annular sector of six enginescale HPNGVs (five passages). In the tests reported here, only one vane was cooled. Contoured sidewalls and downstream deswirl vanes generate periodic boundary conditions, enginerealistic radial pressure gradients, secondary flow features and coolant redistribution. The working section of the facility is shown in Fig. 6. Operating conditions are summarised in Tab. 2 (see also [6-9]).

TABLE 2. OPERATING CONDITIONS OF THE SECTOR FACILITY AND COMPARISON TO APPROXIMATE ENGINE VALUES.

\begin{tabular}{lcc}
\hline \hline Parameter & Sector Facilit: & Engine \\
Inlet mainstream $p_{0}$ & 3.30 bara & $\sim 40$ bara \\
Coolant-to-mainstream PR & $1.02-1.07$ & $\sim 1.03$ \\
Mainstream $T_{0}$ & $330-360 \mathrm{~K}$ & $\sim 1800 \mathrm{~K}$ \\
Coolant $T_{0}$ & $\sim 300 \mathrm{~K}$ & $\sim 900 \mathrm{~K}$ \\
Inlet Tu & $12 \%$ & $12 \%$ \\
Average NGV exit M & 0.90 & 0.90 \\
Average NGV exit Re & $1.2 \times 10^{6}$ & $\sim 1.7 \times 10^{6}$ \\
Coolant Re (based on $0.75 \mathrm{~mm})$ & $\sim 3000$ & $\sim 9000$ \\
Coolant $\gamma_{c}$ & 1.40 & 1.33 \\
Mainstream $\gamma_{m}$ & 1.40 & 1.30 \\
\hline \hline
\end{tabular}




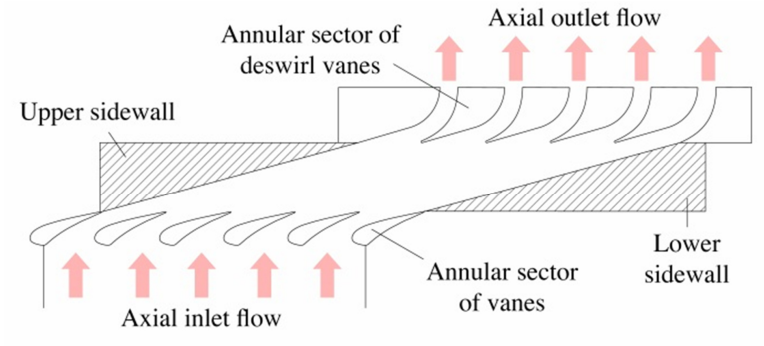

(a) Radial slice of Sector Facility working section.

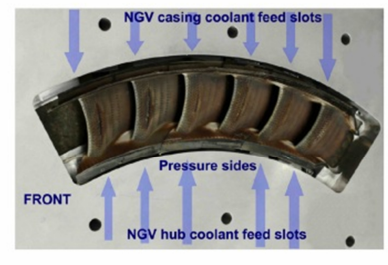

(b) Upstream of vanes; inlet contraction removed.

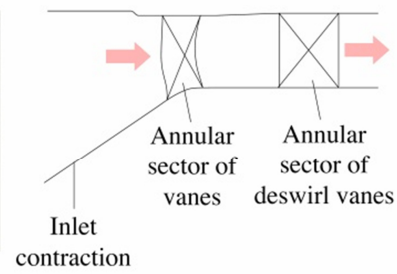

(c) Meridional slice of Sector Facility working section.
FIGURE 6. THE OXFORD UNIVERSITY ANNULAR SECTOR HEAT TRANSFER FACILITY.

During a run (in which the mainstream Mach number distribution is matched to engine conditions), the coolant capacity is measured using a calibrated venturi nozzle. This is typically done at a single $\mathrm{PR}\left(p_{0 c} / p_{0 m}\right)$ per run. The facility is conditioned in such a way that the pressure ratio can be precisely set during a run, but can be modified between tests by changing a limiter in the coolant feed system. By doing so, an overall vane coolant capacity characteristic can be determined (single external Mach number distribution, but many coolantto-mainstream pressure ratios).

\section{METHOD DEMONSTRATION: VANE WITH UNCOUPLED COOLING ROWS (GEOMETRY A)}

We now demonstrate the improved capacity measurement technique using a recent production-engine HPNGV (cast vane pair). We call this geometry $A$. We later demonstrate there is little coupling between cooling rows in geometry A, so this design serves as an example of an uncoupled system.

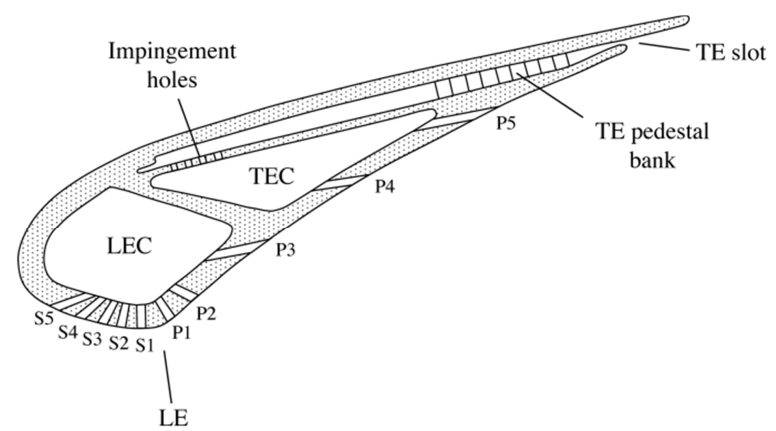

FIGURE 7. SCHEMATIC OF GEOMETRY A (NOT TO SCALE) FROM [6].
A schematic of geometry A is shown in Fig. 7 (photograph in Fig. 2). The vane is fed from both hub and case into a leading-edge compartment (LEC) and trailing-edge compartment (TEC). The LEC feeds film cooling rows S5-P3. The TEC feeds film cooling rows $\mathrm{P} 4, \mathrm{P} 5$ and an impingement system that feeds the TE slot via a pedestal bank. An impingement plate is located in the rear compartment and a midspan baffle plate is located in the front compartment.

\section{Measured Coolant Capacity (Geometry A)}

The coolant capacity measured in the $\operatorname{CCR}\left(\Gamma_{i}^{r}\left(\Pi_{a}\right), \Gamma_{j}^{c}\left(\Pi_{a}\right)\right.$ and $\left.\Gamma^{t}\left(\Pi_{a}\right)\right)$ are now plotted for geometry A. In the CCR all rows exhaust to atmosphere and so capacity is plotted as a function of the ratio $p_{0 c} / p_{\text {atm }}$, where $p_{0 c}$ is the total pressure at vane inlet (average of points $3 \mathrm{~A}$ and $3 \mathrm{~B}$ in Fig. 3), and $p_{\text {atm }}$ is atmospheric pressure.

The cumulative individually measured row capacities $\Gamma_{i}^{r}\left(\Pi_{a}\right)$ are plotted in Fig. 8 (+ markers), in which the measured whole vane capacity $\Gamma^{t}\left(\Pi_{a}\right)(\bullet$ markers $)$ is also shown. Good agreement between the cumulative total of individual measurements, and the overall measurement indicate minimal coupling of the internal system. We now examine this in more detail.

First we compare cumulative capacities measured on a compartmental basis $\Gamma_{j}^{c}\left(\Pi_{a}\right)$ (LEC and TEC), with the measured whole vane capacity $\Gamma^{t}\left(\Pi_{a}\right)$. This comparison is shown in Fig. 9. Excellent agreement between the cumulative (compartmental basis) capacity and the whole vane capacity demonstrates minimal interaction between compartment feeds. In this case the agreement is to within $0.1 \%$. We can say that the compartments in geometry A are effectively uncoupled, and non-interacting.

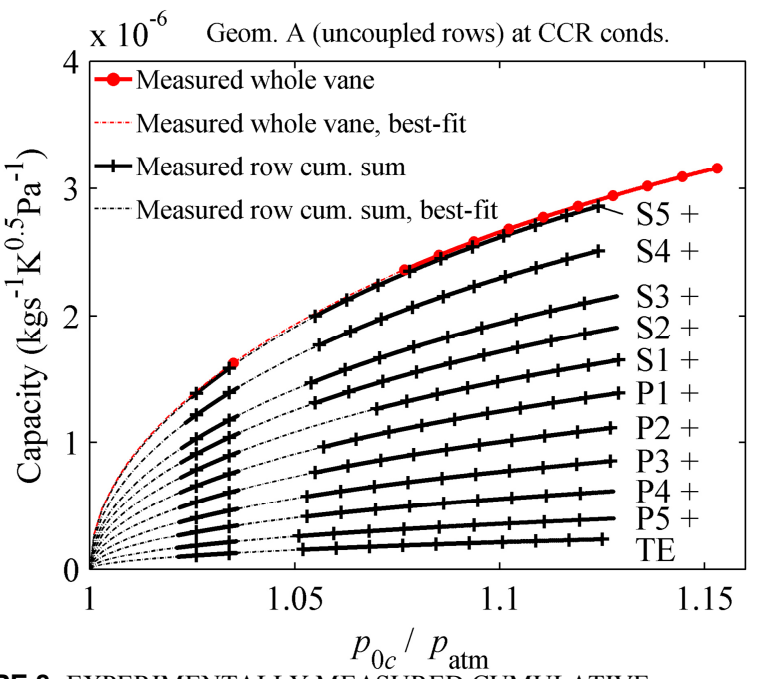

FIGURE 8. EXPERIMENTALLY MEASURED CUMULATIVE INDIVIDUAL ROW CAPACITIES $\Gamma_{i}^{r}\left(\Pi_{a}\right)$ AND WHOLE VANE COOLANT CAPACITY $\Gamma^{t}\left(\Pi_{a}\right)$ FOR GEOMETRY A (ALL ROWS EXHAUSTING TO ATMOSPHERE). 


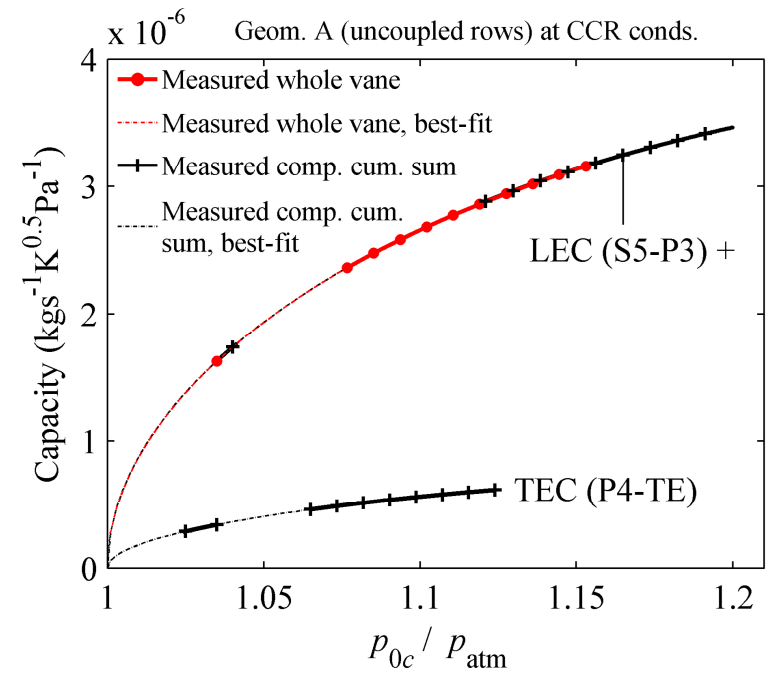

FIGURE 9. EXPERIMENTALLY MEASURED CUMULATIVE COMPARTMENT CAPACITY $\Gamma_{j}^{c}\left(\Pi_{a}\right)$ AND WHOLE VANE COOLANT CAPACITY $\Gamma^{t}\left(\Pi_{a}\right)$ FOR GEOMETRY A.

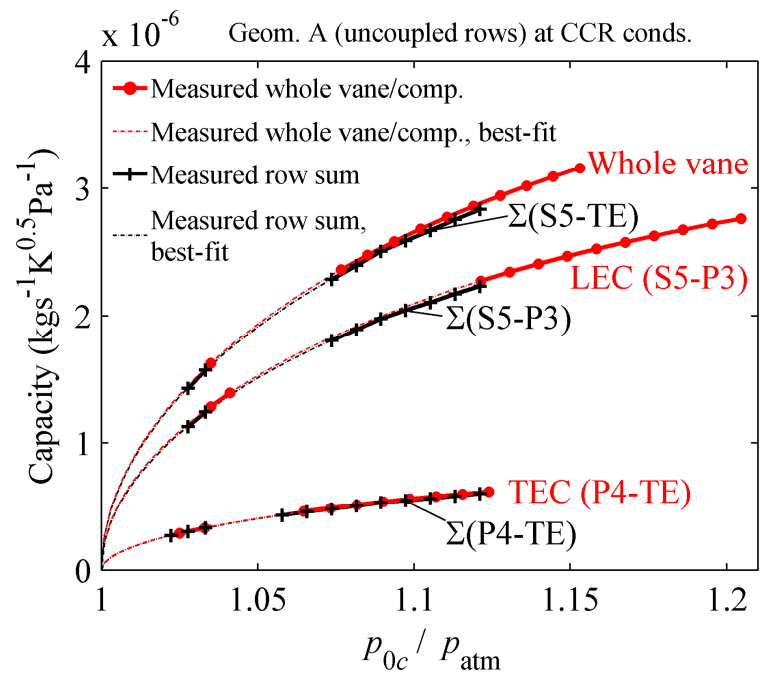

FIGURE 10. EXPERIMENTALLY MEASURED CUMULATIVE COMPARTMENTAL CAPACITY $\Gamma_{j}^{c}\left(\Pi_{a}\right)$ AND WHOLE VANE COOLANT CAPACITY $\Gamma^{t}\left(\Pi_{a}\right)$ COMPARED TO THE SUM OF INDIVIDUAL ROW CAPACITIES $\Gamma_{i}^{r}\left(\Pi_{a}\right)$ WITHIN EACH COMPARTMENT FOR GEOMETRY A.

Now we compare the cumulative individual-row capacity on a per-compartment basis, to the overall capacity measured on a per-compartment basis. This comparison is shown in Fig. 10. Excellent agreement between the sum of row-capacities in the LEC and TEC, and the overall compartment capacities for the LEC and TEC, demonstrates minimal interaction between cooling rows within both the LEC and the TEC. A comparison for the overall vane is also given.

We now consider the results quantitatively. The capacity sum of the individual rows in the TEC (P4, P5 and the TE slot) is within $1.8 \%$ of the overall TEC capacity. Similarly, the capacity sum of the individual rows in the LEC (S5, S4, S3, S2, $\mathrm{S} 1, \mathrm{P} 1, \mathrm{P} 2$ and $\mathrm{P} 3$ ) is within $1.5 \%$ of the overall LEC capacity. Finally, the capacity sum of all individual rows in the vane is within $1.6 \%$ of the whole vane capacity. Good agreement between the sum of individual row capacities and the overall capacity (on a compartment or whole vane basis) shows that the cooling rows in geometry A are relatively uncoupled.

Since the rows in geometry A are relatively uncoupled, the capacity measurement of row $i$ can be considered independently from the capacity of all other rows. We can say that for an uncoupled row, the coolant capacity measurement $\Gamma_{i}^{r}\left(\Pi_{a}\right)$ is equal to the coolant capacity of row $i$ when all rows are blowing $\tilde{\Gamma}_{i}^{r}\left(\Pi_{a}\right)$ :

$$
\tilde{\Gamma}_{i}^{r}\left(\Pi_{a}\right)=\Gamma_{i}^{r}\left(\Pi_{a}\right) .
$$

While this is a trivial result for uncoupled rows, the above equality does not hold for coupled rows because of internal interaction. A more complex method of determining $\tilde{\Gamma}_{i}^{r}$ from $\Gamma_{i}^{r}$ is required for coupled systems. This will be demonstrated using geometry $\mathrm{B}$, later.

CCR capacity measurements are now used to estimate the capacity of each row in the presence of an enginerepresentative external pressure distribution.

\section{Scaling to engine conditions (Geometry A)}

In the CCR, all rows exhaust to atmosphere, compared to the engine where each row exhausts to a different pressure set by the vane aerodynamics. Furthermore, the ratio of specific heats for the coolant at laboratory conditions is $\gamma=1.4$, compared to $\gamma=1.33$ at engine conditions. In this section, these effects are accounted for. Individual row coolant capacities at engine conditions $\tilde{\Gamma}_{i}^{r}\left(\Pi_{e, i}\right)$ are estimated from the individual row coolant capacities measured in the $\mathrm{CCR}, \Gamma_{i}^{r}\left(\Pi_{a}\right) . \Pi_{e, i}$ is the group of

engine (' $e$ ') non-dimensionals related to the $i^{\text {th }}$ row:

$$
\Pi_{e, i}=\left\{\frac{p_{0 c}}{p_{0 m}}, \gamma_{c}, \gamma_{m}, \mathrm{M}_{i}\right\}
$$

where $p_{0 c} / p_{0 m}$ is the ratio of the coolant total pressure at the inlet to the vane to the mainstream total pressure, $\gamma_{c}$ is the coolant ratio of specific heats, $\gamma_{m}$ is the mainstream ratio of specific heats, and $\mathrm{M}_{i}$ is the mainstream Mach number at exit of row $i$. The scaling method is validated using experimental data from the Sector Facility.

The scaling method is now described.

First, the effective areas of each row are estimated from the CCR individual row coolant capacity measurements. The effective areas are presented as functions of local pressure ratio.

To simplify the nomenclature we define a capacity flux function $\zeta(x, y, \gamma)$ (see Appendix A):

$$
\zeta(x, y, \gamma)=x(x y)^{-\frac{\gamma+1}{2 \gamma}} \sqrt{\frac{2 \gamma}{R(\gamma-1)}\left[(x y)^{\frac{\gamma-1}{\gamma}}-1\right]},
$$

where $\gamma$ and $R$ are the ratio of specific heats and specific gas constant of the coolant, $x$ is the ratio of the total pressure immediately upstream of the cooling row to the total pressure at the vane inlet, and $y$ is the ratio of the total pressure at vane inlet to the exhaust static pressure of the cooling row. The product $x y$ is the local pressure ratio across the cooling row (see Appendix A). The effective row area is therefore defined as 
a function of local pressure ratio across the cooling row using the capacity flux function and the capacity of the row:

$$
\tilde{\Gamma}_{i}^{r}=A_{i}^{r}(x y) \cdot \zeta(x, y, \gamma) .
$$

For the simplest case of 1D, compressible, isentropic flow through a-fixed-effective area $A$, at total-to-static $\mathrm{PR} p_{0} / p$, the capacity flux is related to capacity by:

$$
\tilde{\Gamma}_{i}^{r}=A \cdot \zeta\left(1, \frac{p_{0}}{p}, \gamma\right)
$$

Hence, $\zeta(x, y, \gamma)$ is capacity normalised by effective area, i.e., capacity flux. Here we have set $x=1$. For lossy internal systems we have $x<1$, representing a drop in total pressure between the vane inlet, and, for example, a cooling row inlet. This is explained fully in Appendix A.

The effective area of an uncoupled $(x=1)$ cooling row (row $i$ ) at a particular local pressure ratio is estimated from the CCR experiments using eqs. (10) and (13):

$$
A_{i}^{r}\left(\frac{p_{0 c}}{p_{\mathrm{ext}, i}^{r}}\right)=A_{i}^{r}\left(\frac{p_{0 c}}{p_{a t m}}\right)=\frac{\Gamma_{i}^{r}\left(\Pi_{a}\right)}{\zeta\left(1, \frac{p_{0 c}}{p_{\mathrm{atm}}}, \gamma_{a}\right)^{\prime}}
$$

where $p_{\text {ext }, i}^{r}$ is the local exit static pressure of row $i$ and therefore $p_{0 c} / p_{\text {ext }, i}^{r}$ is the local pressure ratio across an uncoupled cooling row. This effective row area incorporates the effect of variable discharge coefficient and pressure loss unique to that row. Later, for coupled systems, we include the additional effect of pressure loss between the vane inlet and row inlet caused by internal row-row interaction, by setting $x<1$ in eq. (15).

The resulting effective areas, $A_{i}^{r}\left(p_{0 c} / p_{\mathrm{ext}, i}^{r}\right)$, are shown in Fig. 11. The effective row areas of geometry $A$ are approximately independent of PR (slight increase as PR increases). The discharge coefficient can of course be estimated from the effective area if the geometric area is known. Geometric area is often difficult to measure, however, especially for non-cylindrical cooling holes, and so it is often more convenient to keep row characterisation in terms of an effective area.

It is assumed that the effective area in the laboratory equals the effective area in the engine. For completeness, we note that Re effects are small (rig coolant capacity (estimated from [3]) is approximately $6 \%$ lower than in the engine), not the prime subject of this paper, and can be considered separately if required. In this analysis we ignore these $\mathrm{Re}$ effects. The coolant capacity of uncoupled row $i$ at engine conditions as a function of local hole pressure ratio $p_{0 c} / p_{\mathrm{ext}, i}^{r}$ and the ratio of specific heats of the coolant at the engine condition $\gamma_{c}$ is given by:

$$
\tilde{\Gamma}_{i}^{r}\left(\frac{p_{0 c}}{p_{\mathrm{ext}, i}^{r}}, \gamma_{c}\right)=A_{i}^{r}\left(\frac{p_{0 c}}{p_{\mathrm{ext}, i}^{r}}\right) \cdot \zeta\left(1, \frac{p_{0 c}}{p_{\mathrm{ext}, i}^{r}}, \gamma_{c}\right) .
$$

The local pressure ratio across a cooling row $p_{0 c} / p_{\text {ext }, i}^{r}$ depends on external pressure (or Mach number) distribution and the coolant-to-mainstream total pressure ratio $p_{0 c} / p_{0 m}$. More usefully, perhaps, we can present the cooling capacity at engine conditions in terms of the coolant-to-mainstream pressure ratio, rather than the local pressure ratio across a cooling hole. To do this we use the following isentropic expression:

$$
\frac{p_{0 c}}{p_{\mathrm{ext}, i}^{r}}\left(\Pi_{i}\right)=\frac{p_{0 c}}{p_{0 m}}\left(1+\frac{\gamma_{m}-1}{2} \mathrm{M}_{i}{ }^{2}\right)^{\frac{\gamma_{m}}{\gamma_{m}-1}}
$$

where $\mathrm{M}_{i}$ is the local mainstream Mach number at the exit of row $i$, and $\gamma_{m}$ is the ratio of specific heats for the mainstream gas, and $\Pi_{i}$ is the group of non-dimensionals:

$$
\Pi_{i}=\left\{\frac{p_{0 c}}{p_{0 m}}, \gamma_{m}, \mathrm{M}_{i}\right\}
$$

This is effectively a change of co-ordinate system, so that $\tilde{\Gamma}_{i}^{r}\left(p_{0 c} / p_{\text {ext }, i}^{r}, \gamma_{c}\right)$ can be recast as $\tilde{\Gamma}_{i}^{r}\left(p_{0 c} / p_{0 m}, \gamma_{c}, \gamma_{m}, \mathrm{M}_{i}\right)$, i.e., $\tilde{\Gamma}_{i}^{r}\left(\Pi_{e, i}\right)$. Therefore for uncoupled rows at engine conditions, eq. (16) can be rewritten using eqs. (11), (17) and (18):

$$
\tilde{\Gamma}_{i}^{r}\left(\Pi_{e, i}\right)=A_{i}^{r}\left(\frac{p_{0 c}}{p_{\mathrm{ext}, i}^{r}}\left(\Pi_{i}\right)\right) \cdot \zeta\left(1, \frac{p_{0 c}}{p_{\mathrm{ext}, i}^{r}}\left(\Pi_{i}\right), \gamma_{c}\right)
$$

For geometries $\mathrm{A}$ and $\mathrm{B}$, individual $\mathrm{M}_{i}$ are given in Tab. 3, estimated using CFD in [9].

Effective area of Geom. A (from exp. only)
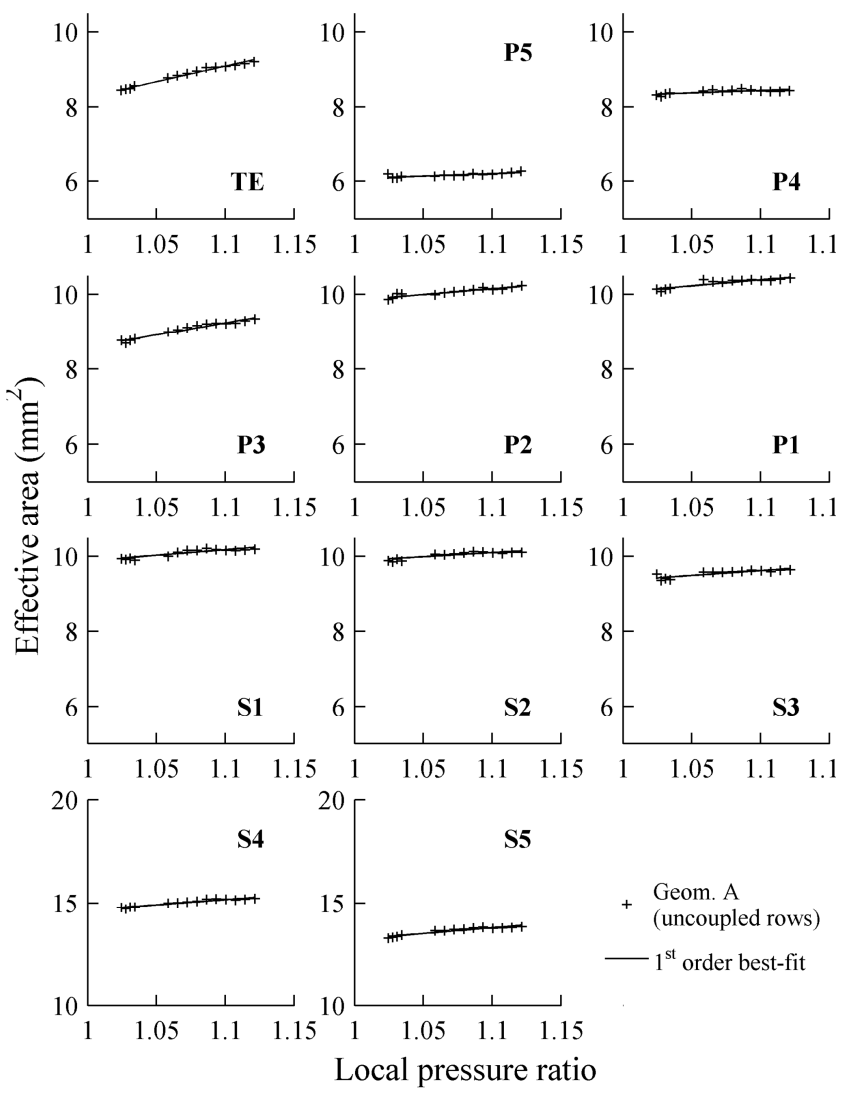

FIGURE 11. EFFECTIVE ROW AREAS $A_{i}^{r}$ OF GEOMETRY A AS A FUNCTION OF LOCAL PRESSURE RATIO $p_{0 c} / p_{\text {ext }, i}^{r}$. 
TABLE 3. ENGINE AND SECTOR FACILITY EXHAUST MACH NUMBER DISTRIBUTION $M_{i}$ FOR GEOMETRY A AND B, INFERRED FROM [9].

\begin{tabular}{ccccccc}
\hline \hline Row & $\mathrm{S} 5$ & $\mathrm{~S} 4$ & $\mathrm{~S} 3$ & $\mathrm{~S} 2$ & $\mathrm{~S} 1$ & $\mathrm{P} 1$ \\
$\mathrm{M}_{i}$ & 0.407 & 0.230 & 0.116 & 0.079 & 0.047 & 0.022 \\
\hline Row & $\mathrm{P} 2$ & $\mathrm{P} 3$ & $\mathrm{P} 4$ & $\mathrm{P} 5$ & $\mathrm{TE}$ & \\
$\mathrm{M}_{i}$ & 0.053 & 0.088 & 0.159 & 0.287 & 0.743 & \\
\hline \hline
\end{tabular}

Figure 12 shows the $\tilde{\Gamma}_{i}^{r}\left(\Pi_{e, i}\right)$ predictions for geometry A operating with an engine-representative external Mach number distribution (Tab. 3) for both Sector $\gamma\left(\gamma_{c}=\gamma_{m}=1.4\right)$ and engine $\gamma\left(\gamma_{c}=1.33, \gamma_{m}=1.3\right)$. The experimental total vane coolant capacity measured in the Sector Facility at 19 PRs (blue crosses) is also shown. The capacity curves are shown as a cumulative total).

The shape of the capacity curves in Fig. 12 (note they are presented as a cumulative total, so the difference between two rows gives the individual row characteristic) are different from those in Fig. 8 because individual rows exhaust to different pressures. Even for $p_{0 c} / p_{0 m}=1$, for example, most cooling rows eject flow because local (individual hole exit) static pressures are almost everywhere significantly below the mainstream total pressure. For rows for which $p_{\mathrm{ext}, i}^{r} / p_{0 m}$ is particularly low (S5 and the TE, for example) row capacity is relatively insensitive to $p_{0 c} / p_{0 m}$ in the range $1.000<p_{0 c} / p_{0 m}<1.088$. For these rows, the local driving pressure across the row is so high that variation in $p_{0 c} / p_{0 m}$ has a limited effect on the local driving pressure ratio.

The agreement between the Sector experimental data and CCR-based predictions is very good, giving confidence in the scaling methods defined by eqs. (15)-(17). For completeness, it is worth noting that the difference in $\gamma$ between Sector and engine conditions gives rise to approximately $1.5 \%$ difference in the coolant capacity prediction.

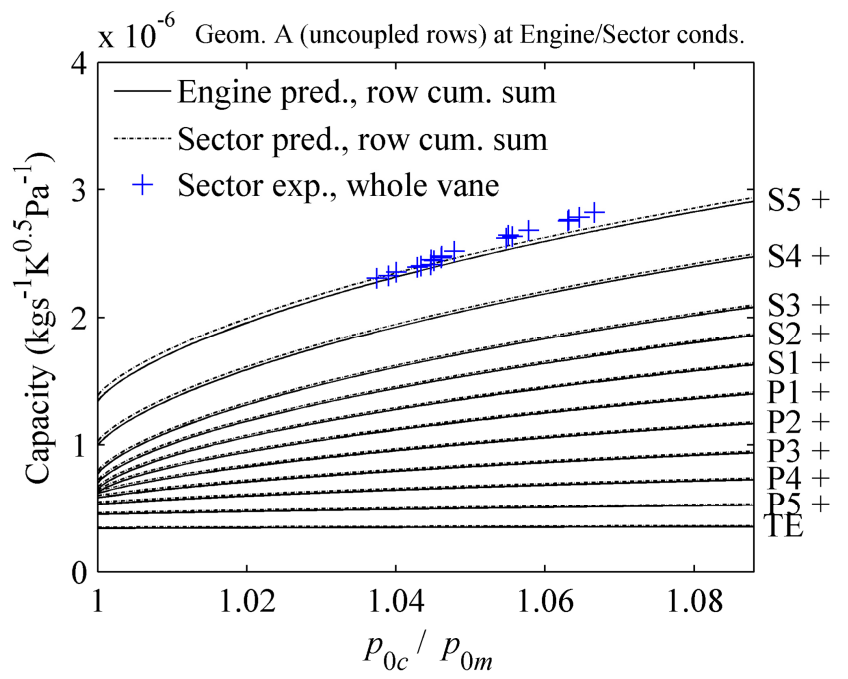

FIGURE 12. CUMULATIVE ROW COOLANT CAPACITY $\tilde{\Gamma}_{i}^{r}\left(\Pi_{e, i}\right)$ PREDICTION WITH ENGINE-REPRESENTATIVE EXTERNAL PRESSURE DISTRIBUTION VALIDATED USING SECTOR FACILITY EXPERIMENTS AT ENGINE-REPRESENTATIVE CONDITIONS (GEOMETRY A).
So far, we have focussed only on turbine vanes with uncoupled cooling rows. We have detailed a fast and repeatable method for estimating individual-row coolant capacity using experimental and analytical methods, and validated these procedures with an experiment at engine-representative conditions. We now examine the more complex problem of vanes with coupled cooling rows.

\section{METHOD DEMONSTRATION: VANE WITH COUPLED COOLING ROWS (GEOMETRY B)}

Geometry B, shown schematically in Fig. 13 (also Fig. 2), is an example of a HPNGV cooling system with strongly coupled cooling rows. We demonstrate experimentally that S4 and S5, in particular, have strong coupling. This is because they are fed by a comparatively long narrow duct.

Geometry B is fed from both hub and case into a single internal plenum, shown in Fig. 13. The plenum is conceptually split into two regions, LEC RP and TEC. The region marked LEC RP feeds rows S3-P3. The region marked TEC feeds row $\mathrm{P} 4-\mathrm{P} 5$, the TE slot, and rows S4 and S5 via a lossy reverse pass channel (RPC, for more details see [10]).

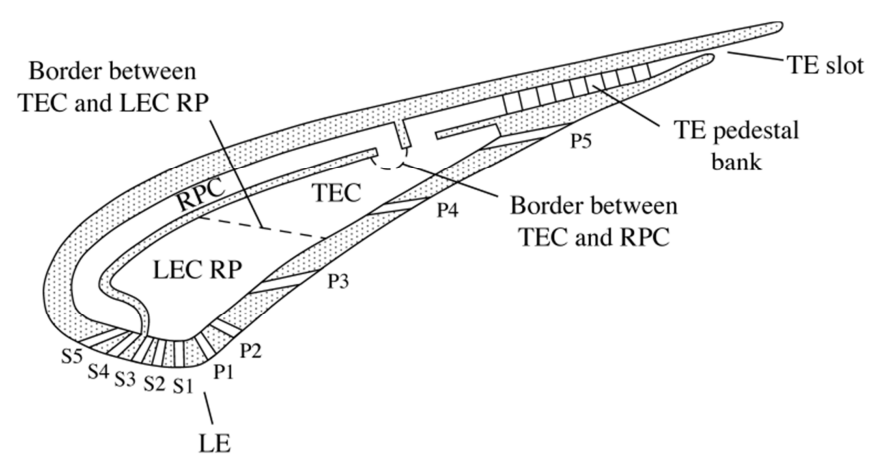

FIGURE 13. SCHEMATIC OF GEOMETRY B (NOT TO SCALE).

\section{Measured Coolant Capacity (Geometry B)}

Experimentally measured (CCR) coolant capacity results for geometry B are now plotted in Figs. 14, 15 and 16.

Cumulative individually measured row capacities $\Gamma_{i}^{r}\left(\Pi_{a}\right)$ are plotted in Fig. 14, and compared to the whole vane capacity $\Gamma^{t}\left(\Pi_{a}\right)$ measurement. The sum of the individually measured row capacities is $15 \%$ lower than the total vane capacity (Fig. 14). Poor agreement between the cumulative total of individual row measurements and the overall vane measurement indicates significant coupling of the internal system. At first the sign of the discrepancy may be surprising (whole vane larger than sum of individual rows). The explanation now follows.

When a particular row (the row of interest) is blocked for the low-differential measurement, the total mass flow rate through the vane is lower than for the corresponding highdifferential measurement, and therefore internal total pressure losses are decreased. This leads to higher total pressure to all rows other than the row of interest (which is blocked) in the low-differential measurement, and higher mass flow rate (for a given system input total pressure) through those holes than in the high-differential measurement. In the method described in this paper, therefore, when the difference between the high- and 
low-differential measurements is taken to determine the capacity of a particular row, the method results in a capacity lower than in the fully coupled state. Nonetheless, for reasons that will be appreciated from earlier sections, the proposed method is believed to be an improvement over earlier techniques.

Now we compare cumulative capacities measured on a compartmental basis $\Gamma_{j}^{c}\left(\Pi_{a}\right)$ (LEC and TEC), with the measured whole vane capacity: Fig. 15. Excellent agreement between the cumulative (compartmental basis) capacity and the whole vane capacity demonstrates minimal interaction between compartment feeds. The agreement is to within $0.5 \%$. We can say that the compartments in geometry $\mathrm{B}$ are effectively uncoupled, and non-interacting. That is, rows grouped by conceptual regions LEC RP, TEC and RPC do not interact with each other.

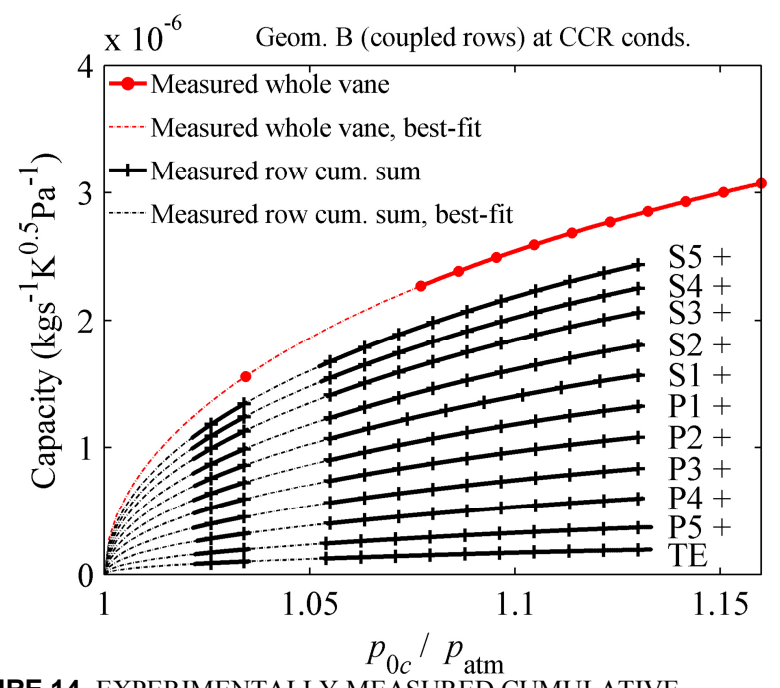

FIGURE 14. EXPERIMENTALLY MEASURED CUMULATIVE INDIVIDUAL ROW CAPACITY $\Gamma_{i}^{r}\left(\Pi_{a}\right)$ AND WHOLE VANE COOLANT CAPACITY $\Gamma^{t}\left(\Pi_{a}\right)$ FOR GEOMETRY B (ALL ROWS EXHAUSTING TO ATMOSPHERE).

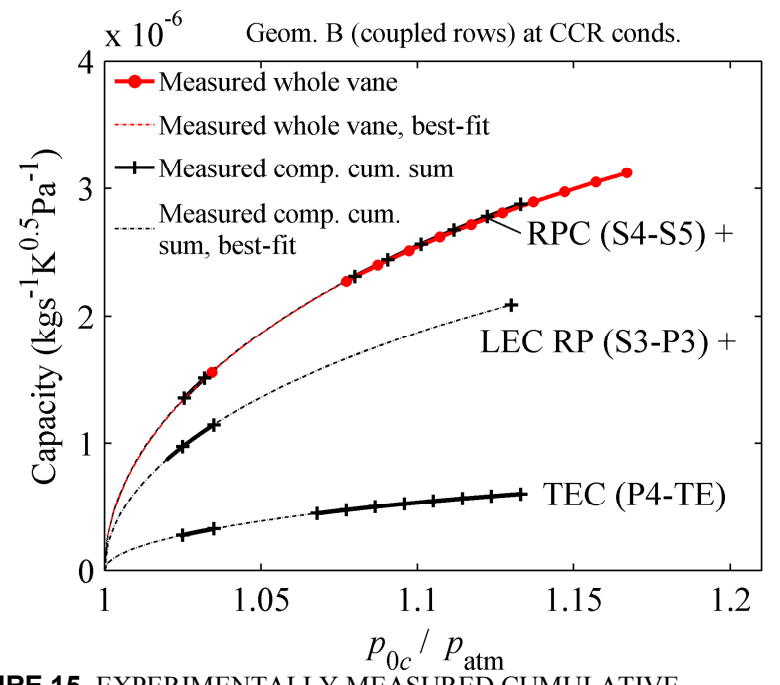

FIGURE 15. EXPERIMENTALLY MEASURED CUMULATIVE COMPARTMENT CAPACITY $\Gamma_{j}^{c}\left(\Pi_{a}\right)$ AND WHOLE VANE COOLANT CAPACITY $\Gamma^{t}\left(\Pi_{a}\right)$ FOR GEOMETRY B.

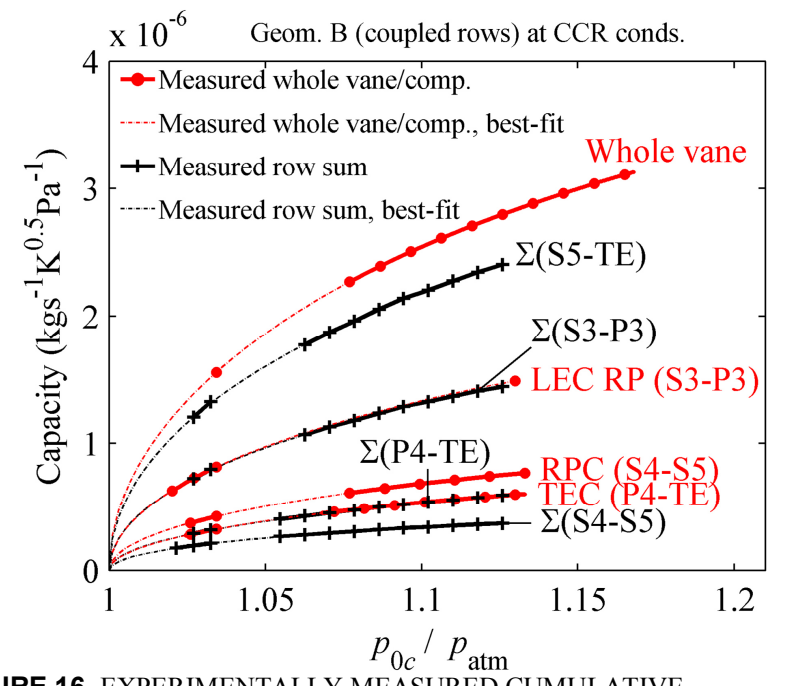

FIGURE 16. EXPERIMENTALLY MEASURED CUMULATIVE COMPARTMENTAL CAPACITY $\Gamma_{j}^{c}\left(\Pi_{a}\right)$ AND WHOLE VANE COOLANT CAPACITY $\Gamma^{t}\left(\Pi_{a}\right)$ COMPARED TO THE SUM OF INDIVIDUAL ROW CAPACITIES $\Gamma_{i}^{r}\left(\Pi_{a}\right)$ WITHIN EACH COMPARTMENT FOR GEOMETRY B.

Now we compare the cumulative individual-row capacity on a per-compartment basis, to the overall capacity measured on a per-compartment basis: Fig. 16. Excellent agreement between the sum of row-capacities in conceptual compartments LEC RP and TEC, and the overall compartment capacities for conceptual compartments LEC PR and TEC, demonstrates minimal interaction between cooling rows within conceptual compartments the LEC RP and TEC. In contrast the sum of capacities for individual rows S4 and S5 is approximately 50\% smaller than the RPC overall compartmental capacity. This shows strong interaction in the RPC compartment. This interaction is the predominant coupling mechanism for geometry $\mathrm{B}$, and accounts for the difference in the cumulative individually measured row capacities (Fig. 14) and measured whole vane capacity.

By comparing individual row capacities and compartmental capacities, we develop a technique to determine internal loss coefficients. Determination of these coefficients (from the data already presented) allows different flow conditions (individual row, compartmental and whole vane) to be reconciled, and allows precise prediction of row capacity at engine conditions.

\section{Network Flow Model to Determine Internal Loss Coefficient and Effective Row Areas (Geometry B)}

In order to calculate loss coefficients from the CCR measurements, it necessary to develop an internal network flow model which includes loss terms that account for row coupling. The form of the network flow model depends on which rows/compartments are most highly coupled, and is deduced from the CCR data. The network flow model is populated using the experimental data from the CCR, and is then used to predict the actual individual-row capacity for any external pressure distribution, any combination of blocked or unblocked rows, and at any ratio of specific heats.

The network flow model used to simulate geometry B is shown in Fig. 17. 


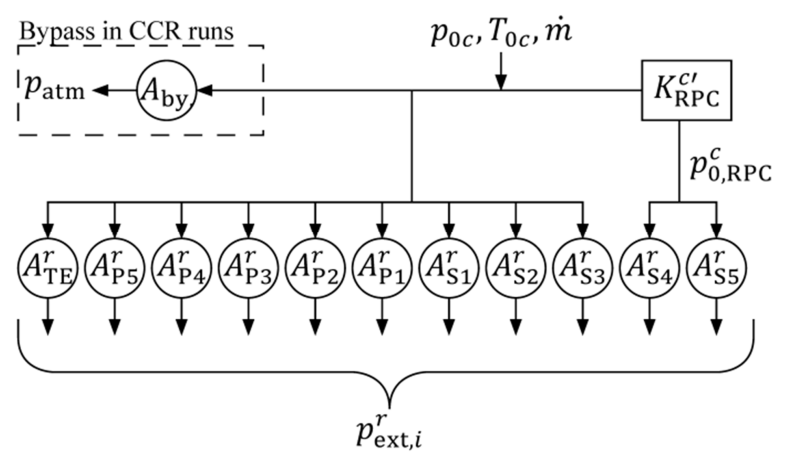

FIGURE 17. NETWORK FLOW MODEL (GEOMETRY B). CIRCLE: COMPRESSIBLE FLOW THROUGH FILM COOLING ROW; SQUARE: INCOMPRESSIBLE LOSS ELEMENT.

The network flow model (the model) includes an internal incompressible loss element, marked by a square (with associated loss coefficient $K_{\mathrm{RPC}}^{c}$ ), and compressible cooling row elements marked by circles, (with capacity characteristics and corresponding underlying area characteristics).

The effective area of the bypass flow $A_{\mathrm{by}}$ is shown in Fig. 17 for completeness, but drops out of the network flow analysis because it is the same for both high- and low-differential runs.

\section{Uncoupled rows}

Experimental data shows that rows S3-TE are highly uncoupled. In the model, therefore, they are fed directly from the vane inlet with no loss elements upstream. The effective areas $A_{i}^{r}$ for rows S3-TE are therefore found using eq. (15). The capacity through the uncoupled rows at engine conditions is given by eq. (19), which we repeat here:

$$
\tilde{\Gamma}_{i}^{r}\left(\Pi_{e, i}\right)=A_{i}^{r}\left(\frac{p_{0 c}}{p_{\mathrm{ext}, i}^{r}}\left(\Pi_{i}\right)\right) \cdot \zeta\left(1, \frac{p_{0 c}}{p_{\mathrm{ext}, i}^{r}}\left(\Pi_{i}\right), \gamma_{c}\right) .
$$

\section{Coupled rows}

Rows S4 and S5 are highly coupled, and in the model are fed via a shared loss element representing the RPC duct. The loss element is represented with a dimensionless loss coefficient $K_{\mathrm{RPC}}^{c}$ :

$$
p_{0 c}-p_{0, \mathrm{RPC}}^{c}=K_{\mathrm{RPC}}^{c} \frac{1}{2} \rho\left(v_{\mathrm{RPC}}^{c}\right)^{2}
$$

where $p_{0 c}$ is the total pressure at vane inlet, $p_{0, \mathrm{RPC}}^{c}$ is the total pressure downstream of the loss element at inlet to S4 or S5, and $\frac{1}{2} \rho\left(v_{\mathrm{RPC}}^{c}\right)^{2}$ is the dynamic head of the fluid in the RPC passage. To simplify the analysis, a dimensional loss coefficient $K_{\mathrm{RPC}}^{C^{\prime}}$ is defined

$$
K_{\mathrm{RPC}}^{c \prime}=\sqrt{\frac{K_{\mathrm{RPC}}^{c} R}{2\left(A_{\mathrm{RPC}}^{c}\right)^{2}}}
$$

where $R$ is the specific gas constant of air and $A_{\mathrm{RPC}}^{c}$ is the flow area of the RPC passage. This allows us to define capacity through the loss element as:

$$
\frac{\dot{m}_{\mathrm{RPC}}^{c} \sqrt{T_{0 c}}}{p_{0 c}}=\frac{1}{K_{\mathrm{RPC}}^{c \prime}} \sqrt{1-\frac{p_{0, \mathrm{RPC}}^{c}}{p_{0 c}}}
$$

where $\dot{m}_{\mathrm{RPC}}^{c}$ is the mass flow rate through the loss element and $T_{0 c}$ is the total temperature at vane inlet.

Flow through each row is defined in terms of a pressureratio-dependent effective area $A_{i}^{r}(x y)$ and the $1 \mathrm{D}$, isentropic, compressible mass flow relation in eq. (13). $x y$ is the local pressure-ratio across the cooling row. For the uncoupled rows $A_{i}^{r}(x y)=A_{i}^{r}\left(p_{0 c} / p_{\mathrm{ext}, i}^{r}\right)$, since $x=1$ in eq. (13). For the coupled rows $\mathrm{S} 4$ and $\mathrm{S} 5, A_{i}^{r}(x y)=A_{i}^{r}\left(p_{0, \mathrm{RPC}}^{c} / p_{\mathrm{ext}, i}^{r}\right)$, since rows S4 and S5 are strongly coupled, and we have $x<1$ in eq. (13) (see derivation in Appendix A) representing a drop in total pressure between the vane inlet and a cooling row inlet. Equation (20) is modified so for S4 and S5 operating at ambient conditions,

$$
\tilde{\Gamma}_{i}^{r}\left(\Pi_{a}\right)=A_{i}^{r}\left(\frac{p_{0, \mathrm{RPC}}^{c}}{p_{0 c}}\left(\Pi_{a}\right) \cdot \frac{p_{0 c}}{p_{a t m}}\right) \cdot \zeta\left(\frac{p_{0, \mathrm{RPC}}^{c}}{p_{0 c}}\left(\Pi_{a}\right), \frac{p_{0 c}}{p_{a t m}}, \gamma_{c}\right) .
$$

We now wish to determine the functions $A_{i}^{r}$ and $K_{\mathrm{RPC}}^{c \prime}$, the variables that describe the coupled rows $\mathrm{S} 4$ and $\mathrm{S} 5$, where $A_{i}^{r}$ is in eq. (24) and $K_{\mathrm{RPC}}^{c^{\prime}}$ in eq. (23) This requires four experimental runs:

1. High-differential run (bypass and all rows open), $\Gamma_{H}\left(\Pi_{a}\right)$

2. S4 Low-differential run (bypass and all rows open except $\mathrm{S} 4), \Gamma_{\mathrm{S} 4, L}^{r}\left(\Pi_{a}\right)$

3. S5 Low-differential run (bypass and all rows open except $\mathrm{S} 5), \Gamma_{\mathrm{S} 5, L}^{r}\left(\Pi_{a}\right)$

4. RPC Low-differential run (bypass and all rows open except $\mathrm{S} 4$ and $\mathrm{S} 5), \Gamma_{\mathrm{RPC}, H}^{c}\left(\Pi_{a}\right)$

In total, there are six unknowns in the experiments: three functions we wish to determine, $A_{\mathrm{S} 4}^{r}\left(p_{0, \mathrm{RPC}}^{c} / p_{a t m}\right)$, $A_{\mathrm{S} 5}^{r}\left(p_{0, \mathrm{RPC}}^{c} / p_{a t m}\right), \quad K_{\mathrm{RPC}}^{c^{\prime}}\left(p_{0, \mathrm{RPC}}^{c} / p_{0 c}\right) ; \quad$ and three internal pressure ratio functions particular to specific run configurations used in the procedure defined in this paper:

$$
\left[\frac{p_{0, \mathrm{RPC}}^{c}}{p_{0 c}}\left(\Pi_{a}\right)\right]_{H},\left[\frac{p_{0, \mathrm{RPC}}^{c}}{p_{0 c}}\left(\Pi_{a}\right)\right]_{S 4, L},\left[\frac{p_{0, \mathrm{RPC}}^{c}}{p_{0 c}}\left(\Pi_{a}\right)\right]_{S 5, L}
$$

where H, S4,L and S5,L correspond to experiments (1), (2) and (3) above.

Six simultaneous equations in the six unknowns are written by considering mass flow continuity in the network flow model for each experimental scenario. In this way, the constants in the network flow model constants are found by running a number of experiments where the outlet boundary conditions are changed. In this case, the rows S4 and S5 are either both blocked, one blocked, or neither blocked. The six equations are derived using the following continuity arguments: 
1. The difference in measured capacity between the highdifferential run and the S4 low-differential run is equal to the difference in capacity through the RPC loss element between those two runs;

2. The difference in measured capacity between the highdifferential run and the S5 low-differential run is equal to the difference in capacity through the RPC loss element between those two runs;

3. The difference in measured capacity between the highdifferential run and the RPC low-differential run is equal to capacity through the RPC loss element in the high differential run;

4. In the high-differential run, the sum of the S4 capacity and S5 capacity equals the capacity through the RPC loss element;

5. In the S4 low-differential run, the S5 capacity equals the capacity through the RPC loss element;

6. In the S5 low-differential run, the S5 capacity equals the capacity through the RPC loss element.

Continuity equations were then written using eq. (23) and (24). The equations are given in Appendix B. The six unknown functions were uniquely determined by solving the simultaneous equations across the range of PRs for which capacity data $\left(\Gamma_{H}, \Gamma_{\mathrm{S} 4, L}^{r}, \Gamma_{\mathrm{S} 5, L}^{r}\right.$ and $\left.\Gamma_{\mathrm{RPC}, L}^{c}\right)$ was measured. The results for geometry B are shown in Figs. 18 and 19

The effective row areas in geometry B are approximately independent of PR, although they generally decrease slightly as PR decreases. Figure 19 shows that the loss coefficient in the $\mathrm{RPC}$ is approximately 2.5 . A slight decrease in $K_{\mathrm{RPC}}^{c}$ with PR is observed in the range tested $(\sim 9 \%)$ which is consistent with studies carried out on turbulator ribs (e.g., [11] and [12] which show friction factor reductions with increased $\mathrm{Re}$ ).

$K_{\mathrm{RPC}}^{c}$ represents the loss in locations where S4 and S5 flow interact, i.e., within the RPC passage before S5. It does not represent row-specific loss, e.g., the loss in the RPC between rows S5 and S4, which is specific to S4. The effect of this loss is implicitly incorporated into the effective area of S4.

In this technique, determination of the internal loss coefficient $K_{\mathrm{RPC}}^{c}$ is possible without internal pressure measurement probes.

\section{Predicted Coolant Capacity when All Rows Exhaust to Atmosphere (Geometry B)}

Since all constants in the network flow model are now known, we can use the model to predict the individual-row cooling capacity of geometry B when all rows exhaust to atmosphere. In the experiments used in this method, the row of interest is covered during the low-differential run. This modifies the pressure loss within the vane, with the result that the capacities of coupled rows are lower than in the case when all rows are blowing (the direction of the change has been discussed in detail in the context of Figs. 14 and 16).

Since rows S3-TE in geometry B are uncoupled, the capacity of these rows is unchanged when all rows exhaust to atmosphere. In our method they can be evaluated directly, eq. (10). To evaluate the capacities of coupled rows S4 and S5
Effective area of Geom. B (from exp. and pred.)
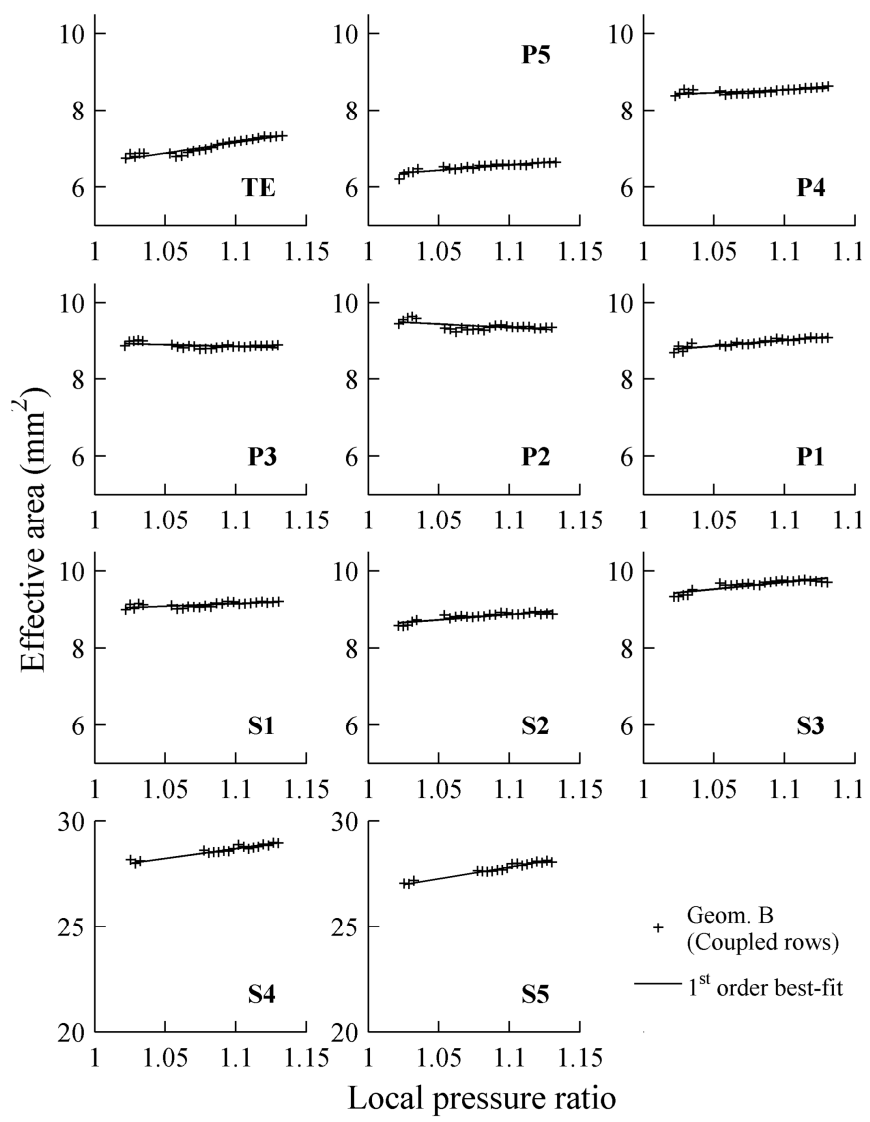

FIGURE 18. EFFECTIVE ROW AREAS $A_{i}^{r}$ OF GEOMETRY B AS A FUNCTION OF LOCAL PRESSURE RATIO. FOR TE-S3, LOCAL PRESSURE RATIO $=p_{0 c} / p_{\text {ext }, i}^{r}$; FOR S5 AND S4, LOCAL PRESSURE $\mathrm{RATIO}=p_{0, \mathrm{RPC}}^{c} / p_{\text {ext }, i}^{r}$.

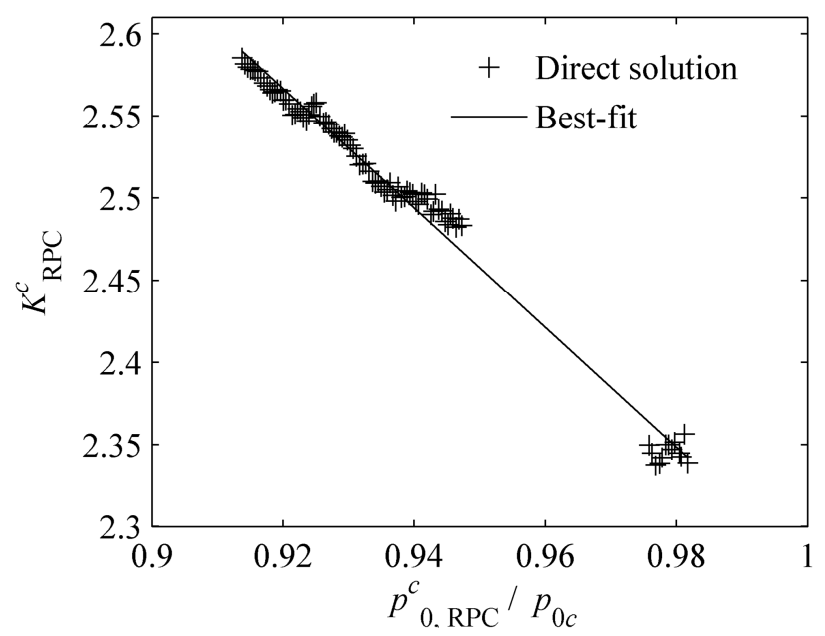

FIGURE 19. DIMENSIONLESS LOSS COEFFICIENT $K_{\mathrm{RPC}}^{c}$ OF GEOMETRY B AS A FUNCTION OF PRESSURE RATIO ACROSS RPC. NOMINAL FLOW AREA $A_{\mathrm{RPC}}^{c}=50 \mathrm{MM}^{2}$. 
when all rows exhaust to atmosphere, we first evaluate the function $\left(p_{0, \mathrm{RPC}}^{c} / p_{0 c}\right)\left(\Pi_{a}\right)$ by solving:

$$
\begin{aligned}
& {\left[A_{\mathrm{S} 4}^{r}\left(\frac{p_{0, \mathrm{RPC}}^{c}}{p_{0 c}}\left(\Pi_{a}\right) \cdot \frac{p_{0 c}}{p_{a t m}}\right)+A_{\mathrm{S} 5}^{r}\left(\frac{p_{0, \mathrm{RPC}}^{c}}{p_{0 c}}\left(\Pi_{a}\right) \cdot \frac{p_{0 c}}{p_{a t m}}\right)\right]} \\
& \cdot \zeta\left(\frac{p_{0, \mathrm{RPC}}^{c}}{p_{0 c}}\left(\Pi_{a}\right), \frac{p_{0 c}}{p_{\mathrm{atm}}}, \gamma_{a}\right) \cdot K_{\mathrm{RPC}}^{c c}\left(\frac{p_{0, \mathrm{RPC}}^{c}}{p_{0 c}}\left(\Pi_{a}\right)\right) \\
& \quad=\sqrt{1-\frac{p_{0, \mathrm{RPC}}^{c}}{p_{0 c}}\left(\Pi_{a}\right),}
\end{aligned}
$$

which is derived from continuity through the RPC using eqs. (23) and (24). Then the capacity of S4 and S5 is calculated using eq. (24). The results of this analysis are shown in Figs. 20 and 21. It is shown in Fig. 20 that for a global pressure ratio of $p_{0 c} / p_{\text {atm }}=1.08$, the local pressure ratio across $\mathrm{S} 4$ and $\mathrm{S} 5$ when all rows exhaust to atmosphere is $p_{0, \mathrm{RPC}}^{c} / p_{\mathrm{atm}}=0.95 * 1.08=$ 1.02 , i.e., the RPC passage loses $75 \%$ of the available global driving pressure.

In Fig. 21 the network flow model prediction $\tilde{\Gamma}_{i}^{r}\left(\Pi_{a}\right)$ is presented on a per-row basis, as a cumulate sum (black lines). It is compared to the sum of measured individual row capacities $\sum \Gamma_{i}^{r}\left(\Pi_{a}\right)$ (green line), and the measured whole vane capacity $\Gamma^{t}\left(\Pi_{a}\right)$ (from Fig. 14, red line). The network model prediction is in excellent agreement with the experimentally measured whole vane capacity measurement (top black line and red line), closing the $15 \%$ gap between the sum of measured individual row capacities (green line), and the measured whole vane capacity (red line). This shows that constants have been correctly evaluated using the network flow method. Small discrepancies between the flow model prediction and whole vane capacity measurement could arise if secondary coupling terms were neglected in the block-diagram description of the model.

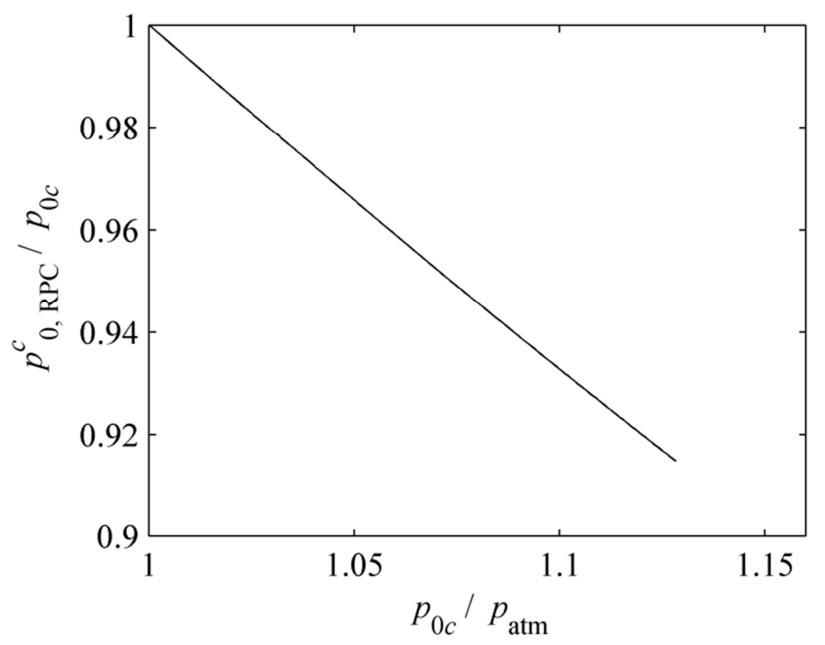

FIGURE 20. TOTAL PRESSURE FEEDING ROWS S4 AND S5 IN COUPLED GEOMETRY B WHEN ALL ROWS EXHAUST TO ATMOSPHERE, PREDICTED USING NETWORK FLOW MODEL.

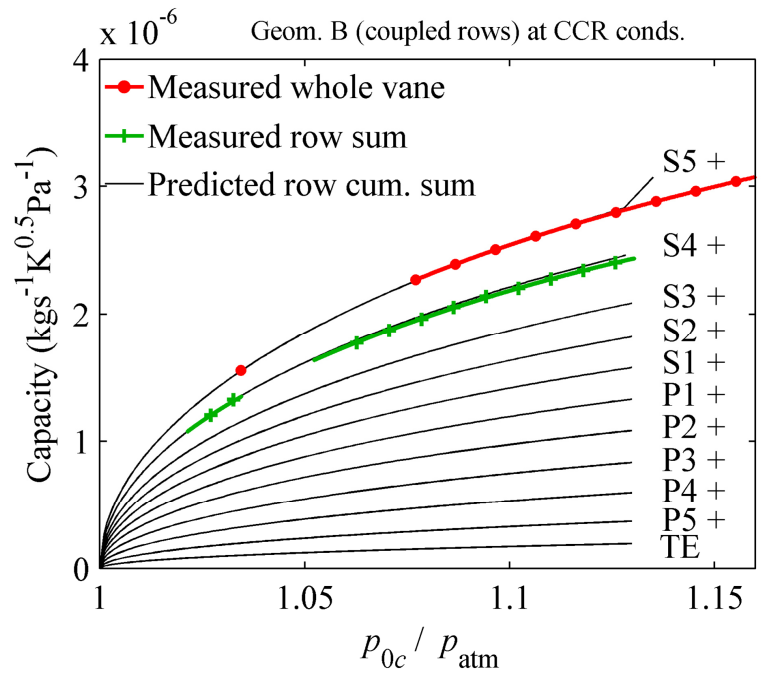

FIGURE 21. NETWORK FLOW MODEL PREDICTED CUMULATIVE ROW COOLANT CAPACITY $\tilde{\Gamma}_{i}^{r}\left(\Pi_{a}\right)$ WHEN ALL ROWS EXHAUST TO ATMOSPHERE, COMPARED TO THE EXPERIMENTAL WHOLE VANE COOLANT CAPACITY $\Gamma^{t}\left(\Pi_{a}\right)$ AND EXPERIMENTAL INDIVIDUALROW CAPACITY SUM $\sum \Gamma_{i}^{r}\left(\Pi_{a}\right)$ (GEOMETRY B).

\section{Scaling to Engine Conditions (Geometry B)}

We now scale Geometry B data from the CCR to engine conditions. In the engine each row exhausts to a different pressure set by the vane aerodynamics, and the coolant ratio of specific heats is approximately $\gamma_{c}=1.33$ (c.f. $\gamma_{c}=1.4$ for the CCR). We now account for these effects, and calculate individual row coolant capacities at engine conditions $\tilde{\Gamma}_{i}^{r}\left(\Pi_{e, i}\right)$ from the individual row coolant capacities measured in the CCR, $\Gamma_{i}^{r}\left(\Pi_{a}\right)$. The scaling method is validated using experimental data from the Sector Facility.

To calculate the coolant capacity $\tilde{\Gamma}_{i}^{r}\left(\Pi_{e, i}\right)$ at engine and Sector Facility conditions we use the network flow model, effective row areas (Fig. 18), RPC loss coefficient (Fig. 19), and the external Mach number distribution (Tab. 3). An important non-dimensional difference between the engine and Sector Facility conditions is that Sector: $\gamma_{c}=\gamma_{m}=1.4$ and engine: $\gamma_{c}=1.33, \gamma_{m}=1.3$.

Since rows S3-TE in geometry B are uncoupled, the capacity characteristics of these rows are unchanged when they exhaust to particular external static pressures. In our method they can be evaluated directly using eq. (19).

To evaluate the capacities of coupled rows S4 and S5 at engine conditions, we first evaluate the unknown function $p_{0, \mathrm{RPC}}^{c} / p_{0 c}$, which depends on the non-dimensional groups

$$
\Pi_{e, R P C}=\left\{\frac{p_{0 c}}{p_{0 m}}, \gamma_{c}, \gamma_{m}, \mathrm{M}_{S 4}, \mathrm{M}_{S 5}\right\} .
$$

$\left(p_{0, \mathrm{RPC}}^{c} / p_{0 c}\right)\left(\Pi_{e, R P C}\right)$ is found by solving: 


$$
\begin{array}{r}
A_{\mathrm{S} 5}^{r}\left(\frac{p_{0, \mathrm{RPC}}^{c}}{p_{0 c}}\left(\Pi_{e, R P C}\right) \cdot \frac{p_{0 c}}{p_{\mathrm{ext}, \mathrm{S} 5}^{r}}\left(\Pi_{S 5}\right)\right) \cdot \zeta\left(\frac{p_{0, \mathrm{RPC}}^{c}}{p_{0 c}}\left(\Pi_{e, R P C}\right), \frac{p_{0 c}}{p_{\mathrm{ext}, \mathrm{S} 5}^{r}}\left(\Pi_{S 5}\right), \gamma_{c}\right) \\
+A_{\mathrm{S} 4}^{r}\left(\frac{p_{0, \mathrm{RPC}}}{p_{0 c}}\left(\Pi_{e, R P C}\right) \cdot \frac{p_{0 c}}{p_{\mathrm{ext}, \mathrm{S} 4}^{r}}\left(\Pi_{S 4}\right)\right) \\
\cdot \zeta\left(\frac{p_{0, \mathrm{RPC}}^{c}}{p_{0 c}}\left(\Pi_{e, R P C}\right), \frac{p_{0 c}}{p_{\mathrm{ext}, \mathrm{S} 4}^{r}}\left(\Pi_{S 4}\right), \gamma_{c}\right)=\frac{\sqrt{1-\frac{p_{0, \mathrm{RPC}}^{c}}{p_{0 c}}\left(\Pi_{e, R P C}\right)}}{K_{\mathrm{RPC}}^{c^{\prime}}\left(\frac{p_{0, \mathrm{RPC}}^{c}}{p_{0 c}}\left(\Pi_{e, R P C}\right)\right)} .
\end{array}
$$

Equation (27) comes from applying continuity in the RPC, as shown in Appendix B. The capacity of S4 and S5 at engine/Sector conditions are then determined using:

$$
\tilde{\Gamma}_{i}^{r}\left(\Pi_{e, R P C}\right)=A_{i}^{r}\left(\frac{p_{0, \mathrm{RPC}}^{c}}{p_{0 c}}\left(\Pi_{e, R P C}\right) \cdot \frac{p_{0 c}}{p_{\mathrm{ext}, \mathrm{i}}^{r}}\left(\Pi_{i}\right)\right) \cdot \zeta\left(\frac{p_{0, \mathrm{RPC}}^{c}}{p_{0 c}}\left(\Pi_{e, R P C}\right), \frac{p_{0 c}}{p_{\mathrm{ext}, \mathrm{i}}^{r}}\left(\Pi_{i}\right), \gamma_{c}\right) .
$$

It is easy to show (discussed later) that there is a range of $p_{0 c} / p_{0 m}$ for which $p_{0, \mathrm{RPC}}^{c} / p_{\text {ext,S4 }}^{r}<1$. This leads to ingestion through row S4. Ingestion is only possible when there is variation in exhaust pressure around the aerofoil (e.g., in the engine or Sector Facility), and does not occur when all rows exhaust to the same pressure (e.g., CCR experiments). Consequently, ingestion need not be considered when populating the network flow model using the CCR data, but must be accounted for when using the model to predict coolant capacity at engine conditions or at Sector Facility conditions.

In the regime $p_{0, \mathrm{RPC}}^{c} / p_{\mathrm{ext}, \mathrm{S} 4}^{r}<1$, reverse-flow though $\mathrm{S} 4$ is modelled using the $1 \mathrm{D}$, isentropic, compressible mass flow relation at a driving pressure ratio $p_{\mathrm{ext}, \mathrm{S} 4}^{r} / p_{0, \mathrm{RPC}}^{c}$. The effective area is evaluated at the pressure ratio $p_{\text {ext,S4 }}^{r} / p_{0, \mathrm{RPC}}^{c}$. This ensures that the capacity of S4 is a continuous function of PR as $p_{0, \mathrm{RPC}}^{c} / p_{\text {ext,S4 }}^{r}$ drops below unity. The continuity equation (27) must also be modified to allow calculation of $p_{0, \mathrm{RPC}}^{c} / p_{0 c}$ when S4 ingests. The details of S4 ingestion modelling are included in Appendix C.

The normalised total pressure characteristics feeding rows S4 and S5 are shown in Fig. 22, for both Engine and Sector conditions of $\gamma_{c}$ and $\gamma_{m}$ (black lines). The S4 ingestion limits (straight red lines) are set by the mainstream Mach number (Tab. 3) and mainstream ratios of specific heats. The ingestion limits are marked by the points where the pressure characteristics (black) cross the ingestion lines (red). These are highlighted with bold points in the figure. To the left hand side of the ingestion limit points, row S5 continues to eject but S4 ingests. Theoretically, there is no flow through the RPC at the point where $\mathrm{S} 4$ ingestion equals $\mathrm{S} 5$ ejection (at $p_{0 c} / p_{0 m}<1$, not shown), and at that point $p_{0, \mathrm{RPC}}^{c} / p_{0 c}=1$.

The whole vane coolant capacity at engine- (uppermost solid black line) and Sector-conditions (uppermost dashed black line) predicted using the network flow model and CCR data are shown in Fig. 23. Experimental data from the Sector are also shown (blue + marks). Excellent agreement between prediction (uppermost dashed black line) and experiment (blue + marks) demonstrate the accuracy of the proposed experimental techniques, and the proposed network model methodology. The cumulative sum of Engine- (solid black lines) and Sector-conditions (dashed black lines) individual row coolant capacities are also shown. The difference between these conditions (caused by dissimilarity in $\gamma_{c}$ and $\gamma_{m}$, and resulting dissimilarity in $\mathrm{M}$ ) is very small (approximately $1.5 \%$ ). The regions of ingestion through row $\mathrm{S} 4$ are marked by red lines (almost identical) in Fig. 23.

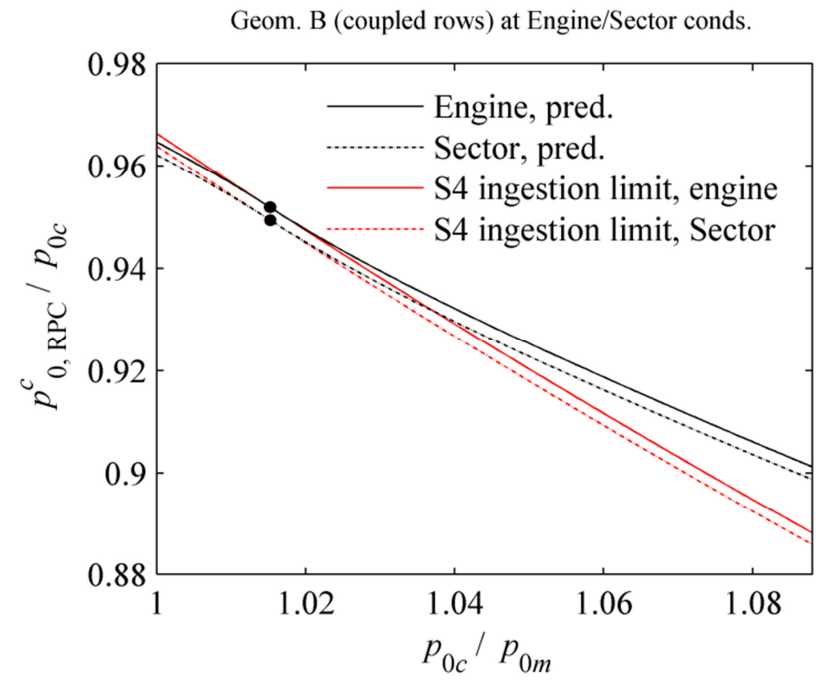

FIGURE 22. NORMALISED TOTAL PRESSURE FEEDING ROWS S4 AND S5 WITH ENGINE- AND SECTOR-REPRESENTATIVE EXTERNAL PRESSURE DISTRIBUTIONS, PREDICTED USING NETWORK FLOW MODEL (SECTOR: $\gamma_{c}=\gamma_{m}=1.4$; ENGINE: $\gamma_{c}=1.33, \gamma_{m}=1.3$ ): GEOMETRY B.

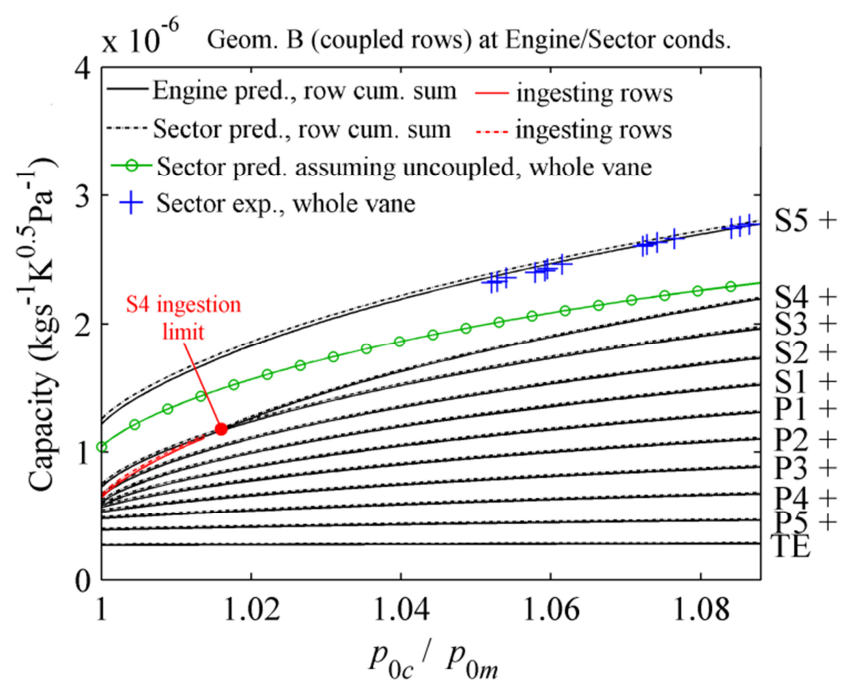

FIGURE 23. CUMULATIVE INDIVIDUAL ROW COOLANT CAPACITY $\left(\tilde{\Gamma}_{i}^{r}\left(\Pi_{e, R P C}\right) \mathrm{S} 5\right.$-S4, $\tilde{\Gamma}_{i}^{r}\left(\Pi_{e, i}\right) \mathrm{S} 3$-TE) WITH ENGINE- AND SECTORREPRESENTATIVE EXTERNAL PRESSURE DISTRIBUTIONS, PREDICTED USING NETWORK FLOW MODEL AND VALIDATED USING SECTOR FACILITY EXPERIMENTS (SECTOR: $\gamma_{c}=\gamma_{m}=1.4$; ENGINE: $\left.\gamma_{c}=1.33, \gamma_{m}=1.3\right)$ : GEOMETRY B. 
For comparative purposes, the whole vane Sectorconditions coolant capacity prediction conducted assuming that S4 and S5 are uncoupled (i.e., using only the data from Fig. 14 and no network flow model) is shown as a green line. This inadequate prediction differs by $\sim 15 \%$ from both the measured data (blue + marks) and the prediction using an adequate method (uppermost dashed black line). This demonstrates the necessity of using both accurate individual-row data, and a network flow model of the type proposed.

\section{CONCLUSIONS}

An experimental method and accompanying scaling theory for accurately evaluating the individual-row coolant capacity characteristics (i.e. effective row areas as a function of pressure ratio) of engine components has been presented. The experimental technique and scaling theory have been validated using extensive experimental data which have been presented.

Experiments are conducted as differential mass flow rate measurements, with respect to a condition which has the closest internal coupling as possible to the engine condition. This minimises later scaling requirements. Variable bypass flow allows a single flow meter to be used across a very wide range of conditions. This significantly reduces the experimental complexity. A method is used whereby the bypass flow does not need to be independently measured.

The accompanying scaling methodology allows experimental results to be accurately scaled between benchtop, high-speed experiment, and full engine conditions. The scaling methodology uses a network flow model (block-diagram) description of the engine component, which defines a system of equations that correctly scale internal coupling terms (internal losses) between different conditions. A model of this type is a minimum requirement to scale accurately between these conditions. A secondary benefit of the network model is that internal duct losses are accurately characterised without the requirement for intrusive instrumentation.

The experimental techniques and associated scaling methodology have been demonstrated using two HPNGVs, which represent examples of relatively coupled (high internal loss coefficients) and relatively uncoupled (low internal loss coefficients) cooling systems. We show that in the case of the coupled system in particular, errors in scaling between benchtop and high-speed environment can be as high as $15 \%$ of the overall vane mass flow rate when simpler techniques are used. Significantly improved accuracy justifies the additional complexity of the network model.

More complex cooling systems than those presented here (in which compartments and rows interact) have also been successfully characterised using these techniques. The methodologies therefore have wide applicability to complex systems with significant internal loss terms. The number of network flow equations increases non-linearly with the degree of coupling, but the basic building blocks remain the same as those described in this paper.

A particular application of the techniques, which was one motivation for the present study, is for predicting individualrow cooling mass flows in additively manufactured HP NGVs. These are of increasing interest to the research community, but there is often considerable uncertainty about geometric row areas, internal surface roughness, and internal pressure losses, leading to research output in which mass flows are insufficiently characterized.

\section{ACKNOWLEDGEMENT}

The support of Rolls-Royce plc is gratefully acknowledged.

\section{NOMENCLATURE}

\section{Abbreviations}

A Geometry with uncoupled film cooling rows

B Geometry with coupled film cooling rows

CCR Coolant Capacity Rig, University of Oxford

comp. Compartment

conds. Conditions

cum. Cumulative

exp. Experimental

Geom. Geometry

HPNGV High pressure nozzle guide vane

LE Leading-edge

LEC Leading-edge compartment, containing rows S5, S4, S3, S2, S1, P1, P2 and P3

LEC RP Leading-edge compartment in reverse-pass geom. B, containing rows $\mathrm{S} 3, \mathrm{~S} 2, \mathrm{~S} 1, \mathrm{P} 1, \mathrm{P} 2$ and $\mathrm{P} 3$

$\mathrm{P} 1$...P5 Pressure-side film cooling rows

PR Pressure ratio

pred. Prediction

rows Film cooling rows

RPC Reverse-pass compartment in geom. B, containing rows $\mathrm{S} 5$ and $\mathrm{S} 4$

S1...S5 Suction-side film cooling rows

Sector Annular Sector Heat Transfer Facility, Oxford

TE Trailing-edge/trailing-edge slot

TEC Trailing-edge compartment, containing rows $\mathrm{P} 4, \mathrm{P} 5$ and the TE slot (TE)

\section{Lower-case}

$b_{i} \quad$ Polynomial coefficients for best-fit $\left[\mathrm{kg}^{2} \mathrm{~s}^{-2} \mathrm{~K} \mathrm{~Pa}^{-2}\right]$

$d \quad$ Charactersistic length [m]

$d_{1} \quad$ CCR venturi meter nominal throat diameter $(5.67 \mathrm{~mm})$

$i \quad$ Row index

j Compartment index

$\dot{m} \quad$ Mass flow rate $\left[\mathrm{kg} \mathrm{s}^{-1}\right]$

$\tilde{m}_{i}^{r} \quad$ Mass flow rate through row $i$ when all rows are blowing $\left[\mathrm{kg} \mathrm{s}^{-1}\right]$

$\dot{m}_{j}^{c} \quad$ Mass flow rate through comapartment $j\left[\mathrm{~kg} \mathrm{~s}^{-1}\right]$

$p \quad$ Static pressure $[\mathrm{Pa}]$

$p_{2} \quad$ Static pressure at position 2 in $\mathrm{CCR}[\mathrm{Pa}]$

$p_{0} \quad$ Total pressure $[\mathrm{Pa}]$

$p_{0 c} \quad$ Total pressure at vane inlet $[\mathrm{Pa}]$

$p_{0 m} \quad$ Total pressure of mainstream [Pa]

$p_{01}, p_{03}$ Total pressure at positions 1 and 3 in CCR [Pa]

$p_{\text {ext }, i}^{r} \quad$ Exhaust pressure of row $i[\mathrm{~Pa}]$

$p_{0, j}^{c} \quad$ Total pressure within compartment $j[\mathrm{~Pa}]$

$p_{\text {atm }} \quad$ Atmospheric pressure $[\mathrm{Pa}]$

$v_{j}^{c} \quad$ Velocity within compartment $j\left[\mathrm{~m} \mathrm{~s}^{-1}\right]$

$x \quad$ Ratio of total pressure immediately upstream of a cooling row to the total pressure at vane inlet 
$y \quad$ Ratio of total pressure at vane inlet to the exhaust static pressure of a cooling row

\section{Upper-case}

$A, A_{i}^{r} \quad$ Area, effective area of row $i\left[\mathrm{~m}^{2}\right]$

$A_{j}^{c} \quad$ Effective flow area of compartment $j\left[\mathrm{~m}^{2}\right]$

$C_{d} \quad$ Discharge coefficient

$C_{D_{1}} \quad$ Discharge coefficient of CCR venturi meter, based on upstream diameter $D_{1}$

$D_{1} \quad$ Pipe diameter upstream of CCR venturi meter (25.4 $\mathrm{mm}$ )

$K_{j}^{c} \quad$ Dimensionless loss coefficient of compartment $j$

$K_{j}^{c \prime} \quad$ Dimensional loss coefficient of compartment $j$ $\left[\mathrm{kg}^{-1} \mathrm{~s} \mathrm{~K}^{-0.5} \mathrm{~Pa}^{1}\right]$

M Mach number

$\mathrm{M}_{\mathrm{i}} \quad$ Mainstream Mach number at exit of row $i$

$R \quad$ Specific gas constant $\left[\mathrm{J} \mathrm{kg}^{-1} \mathrm{~K}^{-1}\right]$

Re Reynolds number

$\mathrm{Re}_{d} \quad$ Reynolds number, characteristic length $d$

$T_{0} \quad$ Total temperature [K]

$T_{01} \quad$ Total temperature at position 1 in CCR [K]

$\mathrm{Tu} \quad$ Turbulence intensity

\section{Greek}

$\gamma, \gamma_{c}, \gamma_{m}$ Ratio of specific heats, coolant, mainstream

$\gamma_{a} \quad$ Ratio of specific heats at CCR (ambient) conditions

$\Gamma, \Gamma^{t} \quad$ Capacity, whole vane capacity $\left[\mathrm{kg} \mathrm{s}^{-1} \mathrm{~K}^{0.5} \mathrm{~Pa}^{-1}\right]$

$\Gamma_{H} \quad$ High-differential capacity measurement (general) $\left[\mathrm{kg} \mathrm{s}^{-1} \mathrm{~K}^{0.5} \mathrm{~Pa}^{-1}\right]$

$\Gamma_{i}^{r}, \Gamma_{j}^{c} \quad$ CCR capacity measurement of row $i$, compartment $j$ $\left[\mathrm{kg} \mathrm{s}^{-1} \mathrm{~K}^{0.5} \mathrm{~Pa}^{-1}\right]$

$\Gamma_{i, L}^{r}, \Gamma_{i, H}^{r} \quad$ Low-differential, high-differential capacity measurement, row $i\left[\mathrm{~kg} \mathrm{~s}^{-1} \mathrm{~K}^{0.5} \mathrm{~Pa}^{-1}\right]$

$\Gamma_{j, L}^{c}, \Gamma_{j, H}^{c} \quad$ Low-differential, high-differential capacity measurement, compartment $j\left[\mathrm{~kg} \mathrm{~s}^{-1} \mathrm{~K}^{0.5} \mathrm{~Pa}^{-1}\right]$

$\tilde{\Gamma}_{i}^{r} \quad$ Capacity of row $i$ when all rows are blowing $\left[\mathrm{kg} \mathrm{s}^{-1}\right.$ $\mathrm{K}^{0.5} \mathrm{~Pa}^{-1}$ ]

$\Pi_{a} \quad$ Ambient non-dimensional group, $\left\{p_{0 c} / p_{a t m}, \gamma_{a}\right\}$

$\Pi_{e, i} \quad$ Engine non-dimensional group affecting uncoupled row $i,\left\{p_{0 c} / p_{0 m}, \gamma_{c}, \gamma_{m}, \mathrm{M}_{i}\right\}$

$\Pi_{e, R P C} \quad$ Engine non-dimensional group affecting rows in RPC (S4 and S5), $\left\{p_{0 c} / p_{0 m}, \gamma_{c}, \gamma_{m}, \mathrm{M}_{S 4}, \mathrm{M}_{S 5}\right\}$

$\Pi_{i} \quad$ Engine non-dimensional group affecting exhaust pressure of row $i\left\{p_{0 c} / p_{0 m}, \gamma_{m}, \mathrm{M}_{i}\right\}$

$\zeta(x, y, \gamma) \quad$ Capacity flux function $\left[\mathrm{J}^{-0.5} \mathrm{~kg}^{0.5} \mathrm{~K}^{0.5}\right]$

\section{REFERENCES}

[1] Povey, T., 2010, "Effect of Film Cooling on Turbine Capacity," Journal of Turbomachinery, 132.

[2] Horlock, J.H., Watson, D.T., and Jones, T.V., 2001, "Limitations on Gas Turbine Performance Imposed by Large Turbine Cooling Flows," Journal of Turbomachinery, 123.

[3] Rowbury, D., 1998, Discharge Coefficients of Nozzle Guide Vane Film Cooling Holes, DPhil thesis, University of Oxford.

[4] BS EN ISO 9300, 2005, Measurement of gas flows by means of critical Venturi nozzles, BSI, 389 Chiswick High Road, London, W4 4AL.
[5] Povey, T., Sharpe, M. and Rawlinson, A.J., 2011, "Experimental measurements of gas turbine flow capacity using a novel transient technique," Journal of Turbomachinery, 133.

[6] Luque, S., Batstone, J., Gillespie, D.R.H., Povey, T., and Romero, E., 2014, "Full Thermal Experimental Assessment of a Dendritic Turbine Vane Cooling Scheme," Journal of Turbomachinery, 136.

[7] Luque, S., and Povey, T., 2011, "A Novel Technique for Assessing Turbine Cooling System Performance," Journal of Turbomachinery, 133.

[8] Luque, S., Aubry, J., and Povey, T., 2009, “A New Engine-Parts Annular Sector Cascade to Prove NGV Cooling Systems," $8^{\text {th }}$ European Conference on Turbomachinery, Fluid Dynamics and Thermodynamics, Verlag der Technischen Universtat Graz, Graz, Austria, March 23-27, pp.865-878.

[9] Luque Martinez, S.G., 2011, A Fully-Integrated Approach to Gas Turbine Cooling System Research, DPhil thesis, University of Oxford.

[10] Kirollos, B., and Povey, T., 2014, "Reverse-Pass Cooling Systems for Improved Performance," Journal of Turbomachinery, 136.

[11] Han, J.C., and Zhang, Y.M., 1992, "High performance heat transfer ducts with parallel broken and V-shaped broken ribs," Int. J. Heat Mass Transfer, Vol. 35, No. 2, pp. 513-523.

[12] Park, J.S., Han, J.C., Huang, Y., Ou, S., and Boyle, R.J., 1992, "Heat transfer performance comparisons of five different rectangular channels with parallel angled ribs," Int. J. Heat Mass Transfer, Vol. 25, No. 11, pp. 2891-2903.

\section{APPENDICES}

\section{Appendix A: Capacity Flux Function}

A capacity flux function $\zeta(x, y, \gamma)$ has been defined to simplify the nomenclature. The capacity flux function is now described.

In this paper, mass flow through a cooling row $\dot{m}_{i}^{r}$ is referenced to the ideal $1 \mathrm{D}$ isentropic compressible mass flow relation using an effective area that takes into account nonisentropic and real 3D effects. Denoting the total temperature and total pressure at the row throat as $T_{0 u, i}^{r}$ and $p_{0 u, i}^{r}$, respectively, and the static pressure at the throat of the cooling row is $p_{d, i}^{r}$, the compressible mass flow relation for the film cooling row is:

$$
\begin{array}{r}
\frac{\dot{m}_{i}^{r} \sqrt{T_{0 u, i}^{r}}}{p_{0 u, i}^{r}} \\
\quad=A_{i}^{r}\left(\frac{p_{0 u, i}^{r}}{p_{d, i}^{r}}\right) \cdot\left(\frac{p_{0 u, i}^{r}}{p_{d, i}^{r}}\right)^{-\frac{\gamma+1}{2 \gamma}}\left\{\frac{2 \gamma}{R(\gamma-1)}\left[\left(\frac{p_{0 u, i}^{r}}{p_{d, i}^{r}}\right)^{\frac{\gamma-1}{\gamma}}-1\right]\right\}^{\frac{1}{2}},
\end{array}
$$

where the effective area is a function of local pressure ratio $A_{i}^{r}\left(p_{0 u, i}^{r} / p_{d, i}^{r}\right), R$ is the specific gas constant (assumed constant in this analysis) and $\gamma$ is the ratio of specific heats.

It is more useful to reference individual row capacity to a common total temperature and total pressure at vane inlet, because measurements are easier at this location, and also because mass flow continuity can be applied through linear combination of individual row capacity.

By assuming that the coolant total temperature at the cooling row throat is equal to the coolant total temperature at vane inlet $T_{0 c}$, and adding in $p_{0 c}$ terms, eq. (29) becomes: 


$$
\frac{\dot{m}_{i}^{r} \sqrt{T_{0 c}}}{p_{0 c}}=A_{i}^{r}\left(\frac{p_{0 u, i}^{r}}{p_{d, i}^{r}}\right) \cdot \frac{p_{0 u, i}^{r}}{p_{0 c}^{r}}\left(\frac{p_{0 c}}{p_{d, i}^{r}} \frac{r_{0 u, i}^{r}}{p_{0 c}^{r}}\right)^{-\frac{\gamma+1}{2 \gamma}}\left\{\frac{2 \gamma}{R(\gamma-1)}\left[\left(\frac{p_{0 c}}{p_{d, i}^{r}} \frac{p_{0 u, i}^{r}}{p_{0 c}}\right)^{\frac{\gamma-1}{\gamma}}-1\right]\right\}^{\frac{1}{2}},
$$

$p_{0 u, i}^{r} / p_{0 c}$ is the coolant pressure ratio immediately upstream of the row to the vane inlet. For an internal system with no loss, $p_{0 u, i}^{r} / p_{0 c}=1$. For an internal system with loss, $p_{0 u, i}^{r} / p_{0 c}<1$. $p_{0 c} / p_{d, i}^{r}$ is the overall coolant pressure ratio between the vane inlet and cooling row exhaust. becomes

$$
\text { Defining } x=p_{0 u, i}^{r} / p_{0 c} \text { and } y=p_{0 c} / p_{d, i}^{r}, \quad \text { eq. }
$$

$$
\frac{\dot{m}_{i}^{r} \sqrt{T_{0 c}}}{p_{0 c}}=A_{i}^{r}(x y) \cdot x(x y)^{-\frac{\gamma+1}{2 \gamma}}\left\{\frac{2 \gamma}{R(\gamma-1)}\left[(x y)^{\frac{\gamma-1}{\gamma}}-1\right]\right\}^{\frac{1}{2}},
$$

The function on the right hand side appears frequently in this analysis and so to avoid repetition a function $\zeta$ is defined:

$$
\zeta(x, y, \gamma)=x(x y)^{-\frac{\gamma+1}{2 \gamma}}\left\{\frac{2 \gamma}{R(\gamma-1)}\left[(x y)^{\frac{\gamma-1}{\gamma}}-1\right]\right\}^{\frac{1}{2}} .
$$

In terms of $\zeta$, eq. (31) becomes

$$
\frac{\dot{m}_{i}^{r} \sqrt{T_{0 c}}}{p_{0 c}}=A_{i}^{r}(x y) \cdot \zeta(x, y, \gamma)
$$

Since $\dot{m}_{i}^{r} \sqrt{T_{0 c}} / p_{0 c}$ is capacity, and $A_{i}^{r}(x y)$ is an area, the new function $\zeta$ is capacity/area, i.e., capacity flux.

\section{Appendix B: Simultaneous Equations Describing Network Flow Model}

The difference in measured capacity between the highdifferential run and the S4 low-differential run is equal to the difference in capacity through the RPC loss element between those two runs, therefore

$$
\Gamma_{H}\left(\Pi_{a}\right)-\Gamma_{\mathrm{S} 4, L}^{r}\left(\Pi_{a}\right)=\frac{\sqrt{1-\left[\frac{p_{0, \mathrm{RPC}}^{c}}{p_{0 c}}\left(\Pi_{a}\right)\right]_{H}}-\sqrt{1-\left[\frac{p_{0, \mathrm{RPC}}^{c}}{p_{0 c}}\left(\Pi_{a}\right)\right]_{S 4, L}}}{K_{\mathrm{RPC}}^{c^{\prime}}\left(\frac{p_{0, \mathrm{RPC}}^{c}}{p_{0 c}}\left(\Pi_{a}\right)\right)} .
$$

The difference in measured capacity between the highdifferential run and the S5 low-differential run is equal to the difference in capacity through the RPC loss element between those two runs, therefore

$$
\Gamma_{H}\left(\Pi_{a}\right)-\Gamma_{\mathrm{S}, L}^{r}\left(\Pi_{a}\right)=\frac{\sqrt{1-\left[\frac{p_{0 \mathrm{RPC}}^{c}}{p_{0 c}}\left(\Pi_{a}\right)\right]_{H}}-\sqrt{1-\left[\frac{p_{0, \mathrm{RPC}}^{c}}{p_{0 c}}\left(\Pi_{a}\right)\right]_{S 5, L}}}{K_{\mathrm{RPC}}^{c^{\prime}}\left(\frac{p_{0, \mathrm{RPC}}^{c}}{p_{0 c}}\left(\Pi_{a}\right)\right)} .
$$

The difference in measured capacity between the highdifferential run and the RPC low-differential run is equal to capacity through the RPC loss element in the high differential run, therefore

$$
\Gamma_{H}\left(\Pi_{a}\right)-\Gamma_{\mathrm{RPC}, L}^{c}\left(\Pi_{a}\right)=\frac{\sqrt{1-\left[\frac{p_{0, \mathrm{RPC}}^{c}}{p_{0 c}}\left(\Pi_{a}\right)\right]_{H}}}{K_{\mathrm{RPC}}^{c^{\prime}}\left(\frac{p_{0, \mathrm{RPC}}^{c}}{p_{0 c}}\left(\Pi_{a}\right)\right)} .
$$

In the high-differential run, the sum of the S4 capacity and S5 capacity equals the capacity through the RPC loss element, therefore

$$
\begin{gathered}
{\left[A_{S 4}^{r}\left(\frac{p_{0, \mathrm{RPC}}^{c}}{p_{0 c}}\left(\Pi_{a}\right) \cdot \frac{p_{0 c}}{p_{a t m}}\right)+A_{\mathrm{S} 5}^{r}\left(\frac{p_{0, \mathrm{RPC}}^{c}}{p_{0 c}}\left(\Pi_{a}\right) \cdot \frac{p_{0 c}}{p_{a t m}}\right)\right]} \\
\cdot \zeta\left(\left[\frac{p_{0, \mathrm{RPC}}^{c}}{p_{0 c}}\left(\Pi_{a}\right)\right]_{H}, \frac{p_{0 c}}{p_{\mathrm{atm}}}, \gamma_{a}\right)=\frac{\sqrt{1-\left[\frac{p_{0, \mathrm{RPC}}^{c}}{p_{0 c}}\left(\Pi_{a}\right)\right]_{H}}}{K_{\mathrm{RPC}}^{c^{\prime}}\left(\frac{p_{0, \mathrm{RPC}}^{c}}{p_{0 c}}\left(\Pi_{a}\right)\right)} .
\end{gathered}
$$

In the S4 low-differential run, the S5 capacity equals the capacity through the RPC loss element, therefore

$$
\begin{gathered}
A_{\mathrm{S} 4}^{r}\left(\frac{p_{0, \mathrm{RPC}}^{c}}{p_{0 c}}\left(\Pi_{a}\right) \cdot \frac{p_{0 c}}{p_{a t m}}\right) \cdot \zeta\left(\left[\frac{p_{0, \mathrm{RPC}}^{c}}{p_{0 c}}\left(\Pi_{a}\right)\right]_{S 5, L}, \frac{p_{0 c}}{p_{\mathrm{atm}}}, \gamma_{a}\right) \\
=\frac{\sqrt{1-\left[\frac{p_{0, \mathrm{RPC}}^{c}}{p_{0 c}}\left(\Pi_{a}\right)\right]_{S 5, L}}}{K_{\mathrm{RPC}}^{c^{\prime}}\left(\frac{p_{0, \mathrm{RPC}}^{c}}{p_{0 c}}\left(\Pi_{a}\right)\right)} .
\end{gathered}
$$

In the S5 low-differential run, the S5 capacity equals the capacity through the RPC loss element, therefore

$$
\begin{gathered}
A_{\mathrm{S} 5}^{r}\left(\frac{p_{0, \mathrm{RPC}}^{c}}{p_{0 c}}\left(\Pi_{a}\right) \cdot \frac{p_{0 c}}{p_{a t m}}\right) \cdot \zeta\left(\left[\frac{p_{0, \mathrm{RPC}}^{c}}{p_{0 c}}\left(\Pi_{a}\right)\right]_{S 4, L}, \frac{p_{0 c}}{p_{\mathrm{atm}}}, \gamma_{a}\right) \\
=\frac{\sqrt{1-\left[\frac{p_{0, \mathrm{RPC}}^{c}}{p_{0 c}}\left(\Pi_{a}\right)\right]_{S 4, L}}}{K_{\mathrm{RPC}}^{c^{\prime}}\left(\frac{p_{0, \mathrm{RPC}}^{c}}{p_{0 c}}\left(\Pi_{a}\right)\right)} .
\end{gathered}
$$

\section{Appendix C: S4 Ingestion Modelling}

In the regime $p_{0, \mathrm{RPC}}^{c} / p_{\text {ext,S4 }}^{r}<1$, reverse-flow though $\mathrm{S} 4$ is modelled using the $1 \mathrm{D}$, isentropic, compressible mass flow relation at a driving pressure ratio $p_{\mathrm{ext}, \mathrm{S} 4}^{r} / p_{0, \mathrm{RPC}}^{c}$. The effective area is evaluated at the pressure ratio $p_{\mathrm{ext}, \mathrm{S} 4}^{r} / p_{0, \mathrm{RPC}}^{c}$. For $p_{0, \mathrm{RPC}}^{c} / p_{\mathrm{ext}, \mathrm{S} 4}^{r}<1$ we write: 


$$
\begin{aligned}
& \tilde{\Gamma}_{\mathrm{S} 4}^{r}\left(\Pi_{e, R P C}\right) \\
& \quad=-A_{\mathrm{S} 4}^{r}\left(\left[\frac{p_{0, \mathrm{RPC}}^{c}}{p_{0 c}}\left(\Pi_{e, R P C}\right) \cdot \frac{p_{0 c}}{p_{\mathrm{ext}, \mathrm{S} 4}^{r}}\left(\Pi_{S 4}\right)\right]^{-1}\right) \\
& \quad \zeta\left(\left[\frac{p_{0 c}}{p_{\mathrm{ext}, S 4}^{r}}\left(\Pi_{S 4}\right)\right]^{-1},\left[\frac{p_{0, \mathrm{RPC}}^{c}}{p_{0 c}}\left(\Pi_{e, R P C}\right)\right]^{-1}, \gamma_{c}\right) .
\end{aligned}
$$

(40)

The continuity equation (27) must also be modified to allow calculation of $p_{0, \mathrm{RPC}}^{c} / p_{0 c}$ when $\mathrm{S} 4$ ingests. We write:

$$
\begin{aligned}
& A_{S 5}^{r}\left(\frac{p_{0, R \mathrm{PPC}}^{c}}{p_{0 c}}\left(\Pi_{e, R P C}\right) \cdot \frac{p_{0 c}}{p_{\text {ext } \mathrm{s} S 5}^{r}}\left(\Pi_{S 5}\right)\right) \cdot \zeta\left(\frac{p_{0, \mathrm{RPC}}^{c}}{p_{0 c}}\left(\Pi_{e, \mathrm{RPC}}\right), \frac{p_{0 c}}{p_{\mathrm{ext}, S 5}^{r}}\left(\Pi_{S 5}\right), \gamma_{c}\right) \\
& -A_{S 4}^{r}\left(\left[\frac{p_{0, R P C}^{c}}{p_{0 c}^{c}}\left(\Pi_{e, R P C}\right) \cdot \frac{p_{0 c}}{p_{\text {ext } S 4}^{r}\left(\Pi_{S 4}\right)}\right]^{-1}\right) \\
& \cdot \zeta\left(\left[\frac{p_{0 c}}{p_{\text {ext }, S 4}^{r}}\left(\Pi_{S 4}\right)\right]^{-1},\left[\frac{p_{0, R P C}^{c}}{p_{0 c}}\left(\Pi_{e, R P C}\right)\right]^{-1}, \gamma_{c}\right) \\
& =\frac{\sqrt{1-\frac{p_{0, \mathrm{RPC}}^{c}}{p_{0 c}}\left(\Pi_{e, R P C}\right)}}{K_{\mathrm{RPC}}^{c^{\prime}}\left(\frac{p_{0, \mathrm{RPC}}^{c}}{p_{0 c}}\left(\Pi_{e, R P C}\right)\right)} .
\end{aligned}
$$

The capacity of S4 is then found using eq. (40) and capacity of S5 found from eq. (28). 